Draft VERSion August 22, 2018

Preprint typeset using LTEX style emulateapj v. 08/22/09

\title{
SIMULATIONS OF THE MICROWAVE SKY
}

\author{
Neelima Sehgal ${ }^{1}$, Paul Bode ${ }^{2}$, Sudeep Das $^{2,3}$, CARlos HernandeZ-Monteagudo $^{4}$, Kevin Huffenberger $^{5}$, Yen-Ting \\ LIN $^{6}$, JEREMIAH P. OSTRIKER ${ }^{2}$, AND HY TRAC ${ }^{7}$ \\ Draft version August 22, 2018
}

\begin{abstract}
We create realistic, full-sky, half-arcminute resolution simulations of the microwave sky matched to the most recent astrophysical observations. The primary purpose of these simulations is to test the data reduction pipeline for the Atacama Cosmology Telescope (ACT) experiment; however, we have widened the frequency coverage beyond the ACT bands and utilized the easily accessible HEALPix map format to make these simulations applicable to other current and near future microwave background experiments. Some of the novel features of these simulations are that the radio and infrared galaxy populations are correlated with the galaxy cluster and group populations, the primordial microwave background is lensed by the dark matter structure in the simulation via a ray-tracing code, the contribution to the thermal and kinetic Sunyaev-Zel'dovich (SZ) signals from galaxy clusters, groups, and the intergalactic medium has been included, and the gas prescription to model the SZ signals has been refined to match the most recent X-ray observations. The cosmology adopted in these simulations is also consistent with the WMAP 5-year parameter measurements. From these simulations we find a slope for the $Y_{200}-M_{200}$ relation that is only slightly steeper than self-similar, with an intrinsic scatter in the relation of $\sim 14 \%$. Regarding the contamination of cluster SZ flux by radio galaxies, we find for 148 $\mathrm{GHz}(90 \mathrm{GHz})$ only $3 \%(4 \%)$ of halos have their SZ decrements contaminated at a level of $20 \%$ or more. We find the contamination levels higher for infrared galaxies. However, at $90 \mathrm{GHz}$, less than $20 \%$ of clusters with $M_{200}>2.5 \times 10^{14} M_{\odot}$ and $z<1.2$ have their SZ decrements filled in at a level of $20 \%$ or more. At $148 \mathrm{GHz}$, less than $20 \%$ of clusters with $M_{200}>2.5 \times 10^{14} M_{\odot}$ and $z<0.8$ have their SZ decrements filled in at a level of $50 \%$ or larger. Our models also suggest that a population of very high flux infrared galaxies, which are likely lensed sources, contribute most to the SZ contamination of very massive clusters at 90 and $148 \mathrm{GHz}$. These simulations are publicly available and should serve as a useful tool for microwave surveys to cross-check SZ cluster detection, power spectrum, and cross-correlation analyses.

Subject headings: cosmic microwave background - galaxies: clusters: general - galaxies: general - intergalactic medium - large-scale structure of universe - methods: simulations
\end{abstract}

\section{INTRODUCTION}

Simulations of the microwave sky play a crucial role in accurately extracting constraints on cosmology potentially measurable by current and upcoming microwave background observatories, such as the Atacama Cosmology Telescope $(\mathrm{ACT})^{8}$, the South Pole Telescope $(\mathrm{SPT})^{9}$, and the Planck satellite $^{10}$. These surveys will have complex data processing pipelines and analysis strategies, and any artifacts caused by any one of the many analysis steps can best be controlled by simultaneously running simulations of the microwave sky, with known cosmological and astrophysical inputs, through the same analysis pipeline. These microwave background experiments, which will have finer resolution and higher sensitivity than achieved to date, can potentially constrain cosmological parameters via their measured microwave power spec-

\footnotetext{
${ }^{1}$ Kavli Institute for Particle Astrophysics and Cosmology, Stanford, CA 94305

${ }^{2}$ Department of Astrophysical Sciences, Princeton University, Princeton, NJ 08544

${ }^{3}$ Department of Physics, Princeton University, Princeton, NJ 08540

${ }^{4}$ Max Planck Institut fuer Astrophysik, 85741 Garching bei Muenchen, Germany

${ }^{5}$ Department of Physics, University of Miami, Coral Gables, FL 33146

${ }^{6}$ Institute for the Physics and Mathematics of the Universe, University of Tokyo, Japan

${ }^{7}$ Harvard-Smithsonian Center for Astrophysics, Cambridge, MA 02138

${ }^{8} \mathrm{http}: / /$ www.physics.princeton.edu/act/

${ }^{9}$ http://pole.uchicago.edu/

${ }^{10} \mathrm{http} / / / \mathrm{www}$. sciops.esa.int/PLANCK/
}

trum, higher order correlation functions, Sunyaev-Zel'dovich (SZ) cluster detections, and cross-correlation studies of various microwave components. Moreover, these microwave data sets can be combined with surveys at other wavelengths to yield additional constraints. As such, the accurate recovery of known cosmological inputs, through the variety of methods mentioned above, must be tested with simulations. This necessitates high-resolution, cosmological-scale, microwave simulations that include the most accurate astrophysical components as our current observations will allow.

The microwave sky consists primarily of the following components: (1) the primordial microwave background, which is lensed by the intervening structure between the last scattering surface and observers today, (2) the thermal SZ signal from the hot gas in galaxy clusters, groups, and the intergalactic medium, (3) the kinetic SZ signal from the bulk motion of galaxy clusters, groups, and the intergalactic medium, (4) higher-order relativistic corrections to the thermal and kinetic SZ signals, (5) a population of dusty star forming galaxies that emit strongly at infrared wavelengths but have significant microwave emission, (6) a population of galaxies that emit strongly at radio wavelengths, including active galactic nuclei, which also have significant emission at microwave frequencies, and (7) dust, synchrotron, and free-free emission from the Galaxy.

The simulations discussed here include the above components, mapped at six different frequencies: 30, 90, 148, 219, 277, and $350 \mathrm{GHz}$. The frequencies 148, 219, and 277 are the 
central ACT observing frequencies, as these simulations are used by the ACT team to inform their data analysis pipeline. The additional frequencies have been added to widen the applicability of these simulations to other microwave background experiments. The only components listed above that have been excluded from the microwave maps are the Galactic synchrotron and free-free emission. These two components are not expected to be significant at ACT frequencies, and we leave their inclusion to future work. The maps themselves are full-sky HEALPix maps of resolution 0.4 arcminutes (Nside=8192) and consist of full-sky maps of each component separately as well as a combined sky map, at each frequency. These HEALPix maps can be interpolated into flat sky maps using the bilinear interpolation subroutine included in the HEALPix distribution (Górski et al. 2005). In addition, catalogs of all the halos in this simulation and their various properties, as well as catalogs of the individual infrared and radio galaxies that populate the halos, are provided. These simulations are public and can be downloaded from http://lambda.gsfc.nasa.gov/toolbox/tb_cmbsim_ov.cfm

There are of course many simulations of components of the microwave sky (e.g. Gawiser \& Smoot 1997; Metzler 1998; Gawiser et al. 1998; Sokasian et al. 2001; White et al. 2002; da Silva et al. 2004; Diaferio et al. 2005; Motl et al. 2005; Nagai 2006; Hallman et al. 2007; Pfrommer et al. 2007; Roncarelli et al. 2007; Malte Schäfer \& Bartelmann 2007; Leach et al. 2008; Pace et al. 2008; Shaw et al. 2008; Wik et al. 2008; Carbone et al. 2009; Peel et al. 2009). The simulations discussed here include the components mentioned above, in an internally consistent manner, matched to the most recent observations. These simulations have improved upon the previous simulations discussed in Sehgal et al. (2007) in the following ways:

- radio and infrared galaxy populations are clustered and correlated with the galaxy cluster populations

- the primordial microwave background has been lensed by the dark matter structure in the N-body simulation via a ray-tracing code

- the contribution to the kinetic SZ signal from the intergalactic medium has been included, in addition to that from galaxy clusters

- the gas prescription to model the SZ signals has been refined to match the most recent $\mathrm{X}$-ray observations

- maps have been created at a wider range of frequencies to increase the applicability to other microwave background experiments

- standard HEALPix format for the maps has been adopted

We give a brief summary of the key elements of these simulations below, which we expand upon in greater detail throughout $\S 2$ and $\S 3$.

The large-scale structure simulation was carried out using a tree-particle-mesh code (Bode et al. 2000; Bode \& Ostriker 2003), with a simulation volume of $1000 h^{-1} \mathrm{Mpc}$ on a side containing $1024^{3}$ particles. The cosmology adopted is $\left(\Omega_{b}, \Omega_{m}, \Omega_{\Lambda}, h, n_{s}, \sigma_{8}\right)=(0.044,0.264,0.736,0.71$, $0.96,0.80)$, consistent with the WMAP 5-year results
(Komatsu et al. 2009). The mass distribution covering one octant of the full sky was saved, and halos with a friendsof-friends mass above $1 \times 10^{13} M_{\odot}$ and with a redshift below $z=3$ are identified. The octant is then replicated to create a full-sky simulation. Thus the large-scale structure components in these simulations are unique for one octant, while the primary microwave background and Galactic dust are modeled for the full sky.

The primary microwave background has the same cosmology as the large-scale structure and matches the WMAP 5year Internal Linear Combination map (Bennett et al. 2003; Hinshaw et al. 2009) on large-scales $(l<20)$. The microwave background is then lensed by the large-scale structure in the simulation with a ray-tracing code (Das \& Bode 2008).

The Sunyaev-Zel'dovich signal is derived by adding a gas prescription to the $\mathrm{N}$-body halos which assumes a polytropic equation of state and hydrostatic equilibrium. This model, which is described in more detail in Bode et al. (2009), adjusts four free parameters which are calibrated against X-ray gas fractions as a function of temperature from the sample of Sun et al. (2009) and Vikhlinin et al. (2006). These four free parameters are the star formation rate (which depends on the halo mass and redshift), non-thermal pressure support (from cosmic rays or magnetic fields), dynamical energy transfer from dark matter to gas (a depletion factor which lowers the average baryon fraction to less than the cosmic mean), and feedback from active galactic nuclei (which is tied to the star formation rate). This model describes the gas prescription for halos with friends-of-friends mass $>3 \times 10^{13} M_{\odot}$ and $z<3$.

Smaller halos down to $1 \times 10^{13} M_{\odot}$ and the intergalactic medium for $z<3$ are treated with a different method. The velocity dispersion of a given simulation particle is calculated using its neighbors, and this velocity dispersion is related to the pressure (and thus the thermal SZ signal) via a proportionality constant derived from hydrodynamical simulations (Trac et al. 2009). The kinetic SZ is calculated from the lineof-sight momentum of the particles. For the $3<z<10$ Universe, the temperature is assumed to be isothermal at $10^{4} \mathrm{~K}$, and mass density and momentum shells at various redshift slices are used to construct the thermal and kinetic SZ signals.

The infrared source model has six parameters used to populate halos, and is partially based on Righi et al. (2008). This model satisfies the following observational constraints: the COBE/FIRAS background as in Fixsen et al. (1998) is an upper limit on the total infrared intensity, the effective spectral index for the spectral intensity is $\sim 2.6$ between 145 and $350 \mathrm{GHz}$ (Knox et al. 2004; Dunne et al. 2000), source counts are compatible with SCUBA counts at $353 \mathrm{GHz}$ (Coppin et al. 2006), source counts and clustering are compatible with BLAST measured counts and power spectrum (Patanchon et al. 2009; Viero et al. 2009), and the power of the infrared sources is below the point source upper limit derived by ACBAR at $150 \mathrm{GHz}$, fixing $\sigma_{8}$ to the WMAP 5-year measurement (Reichardt et al. 2009).

The model for the radio sources is made by creating a halo occupation distribution and luminosity distribution for radio sources at $151 \mathrm{MHz}$ to match the $151 \mathrm{MHz}$ radio luminosity function (RLF) at $z \sim 0.1 .151 \mathrm{MHz}$ was chosen because at such low frequencies the RLF is dominated by the steep spectrum lobes of the radio sources (as opposed to the flatter spectrum cores) and is thus less sensitive to biases due to orientation effects. Once the $151 \mathrm{MHz}$ RLF is matched at low redshifts, the radio sources are divided into two popu- 
lations, similar to the types I and II of the Fanaroff \& Riley (1974) classification. These are given differing number density evolutions with redshift, and the RLF at higher redshift is matched to observations. We use a relativistic beaming model to separate how much of a source's flux at $151 \mathrm{MHz}$ is from the core as opposed to the lobes, and we adjust the shape of the radio core spectral energy distribution to match source counts at $\nu \gg 151 \mathrm{MHz}$, where the core dominates (Lin et al.2009a).

The Galactic dust emission maps are from the "model 8" prediction from Finkbeiner et al. (1999), which Bennett et al. (2003) show to be a reasonable template for dust emission in the WMAP maps.

In $\$ 2$, we discuss the construction of each component of these simulations and comparison to observations in more detail. In $\$ 3$, we discuss further properties of the simulated maps, and in $\$ 4$ we summarize and conclude.

\section{SIMULATIONS}

\subsection{Primary Unlensed $C M B$}

We follow the method of Sehgal et al. (2007), constructing a primary microwave background map with the observed large-scale structure and small scale structure consistent with theoretical expectations. For large scales, we use the WMAP 5-year Internal Linear Combination (ILC) map (Bennett et al. 2003; Hinshaw et al. 2009). For $l<20$, we take the harmonic coefficients $\left(a_{l m}\right)$ from the ILC map with no modification. At smaller scales, where the ILC map is smoothed significantly, a Gaussian random realization is added, so that the ensemble average power equals the theoretical power spectrum which maximizes the WMAP 5-year likelihood. Harmonic coefficients are computed to $l_{\max }=8192$, and we synthesize a map with HEALPix parameter $N_{\text {side }}=4096\left(0.859^{\prime}\right.$ pixels $)$. Then we convert to a map with HEALPix parameter $N_{\text {side }}=8192$ $\left(0.429^{\prime}\right.$ pixels $)$ using the alter_alm subroutine included in the HEALPix distribution (Górski et al. 2005). The lower resolution $\left(N_{\text {side }}=4096\right)$ map is used to generate the lensed microwave background map (see $\$ 2.3$, as that is more efficient than working with the $N_{\text {side }}=8192$ map directly and results in no significant loss of accuracy. The lensed map is then also converted to an $N_{\text {side }}=8192$ map.

\subsection{Large-Scale Structure Simulation}

A simulation of the large-scale structure of the Universe is performed using the same methods as previously described in Bode et al. (2007) and Sehgal et al. (2007), and we refer the reader to their papers for additional details. Within a periodic box of comoving side length $L=1000 \mathrm{~h}^{-1} \mathrm{Mpc}, 1024^{3}$ dark matter particles are evolved using the Tree-Particle-Mesh (TPM) N-body code (Bode \& Ostriker 2003). The particle mass is $6.82 \times 10^{10} h^{-1} M_{\odot}$ and the gravitational spline softening length is set to $\epsilon=16.276 h^{-1} \mathrm{kpc}$. This simulation has been previously used by Bode et al. (2009) in developing a model for the intracluster medium.

A putative observer at redshift zero is placed at one corner of the periodic box, and the mass distribution along a past light cone, covering one octant (box coordinates $x, y, z>0$ ) of the sky, is saved as the simulation is running. To create full-sky maps this octant is replicated, as described in $\$ 3$.

At each simulation time step, spanning the redshift range $z_{1}>z>z_{2}$, particles are found in a thin spherical shell at the comoving distance $d$ corresponding to the light travel time to this observer, spanning the range $d\left(z_{1}\right)>d>d\left(z_{2}\right)$. For $z<3$ all of these particles are saved to disk and for $z<10$ pixelized

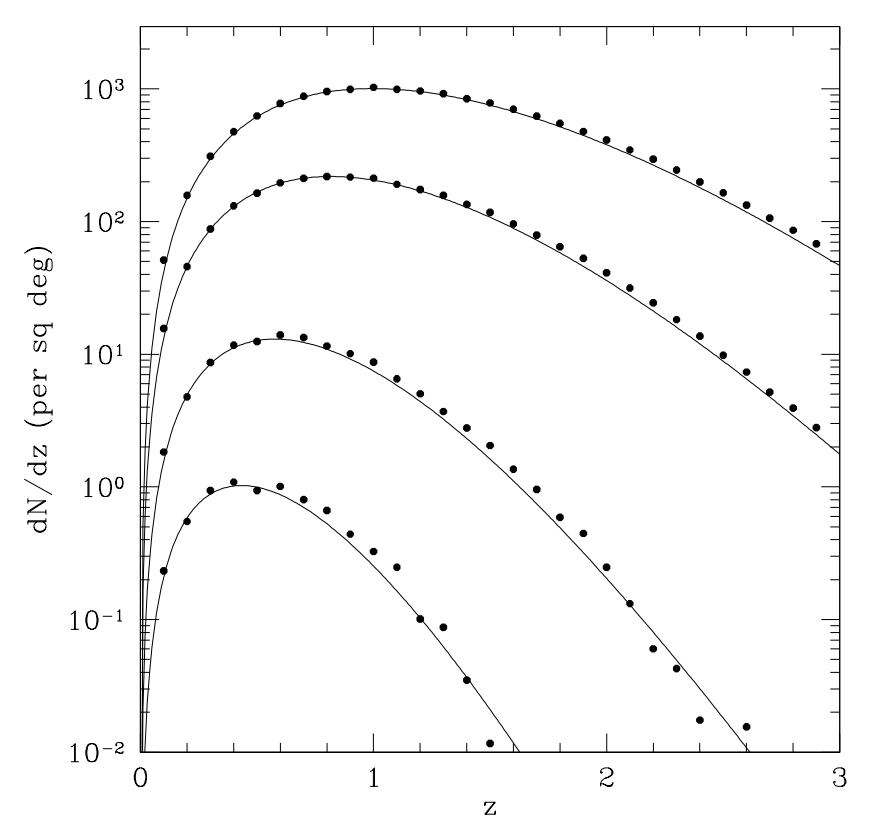

FIG. 1.- The halo abundance above a minimum mass per unit redshift per square degree measured from the large-scale structure simulation (points) is compared with the semi-analytic fitting formula (lines) from Jenkins et al. (2001). From top to bottom, the four sets of points are for the minimum masses $M_{\min }=6.82 \times 10^{12}, 2 \times 10^{13}, 1 \times 10^{14}$, and $3 \times 10^{14} h^{-1} M_{\odot}$.

versions of the shells are saved, as described below. There are 579 such shells in all; the range $\Delta z=z_{1}-z_{2}$ of each shell depends on the time step size set by the TPM code. It decreases from $\Delta z \approx 0.08$ at $z \approx 9$ to $\Delta z \approx 0.03$ at $z \approx 3$, and to $\Delta z<0.005$ for $z<0.8$. For comoving distances larger than the box size, $d>1000 h^{-1} \mathrm{Mpc}$, there can be some duplication in structures as the periodic box is repeatedly tiled. This tiling is done without random rotations to preserve the periodicity and prevent discontinuities in the large-scale structure. However, any repetition is usually at a different redshift and evolutionary state. In cases where a halo or filament appears twice at similar redshifts, it is viewed at two different angles, making each projected image unique.

For all the shells $(z<10)$ particles are subdivided by angular coordinates using the HEALPix scheme for pixelation of a sphere. The projected mass and momentum in each pixel of the shell are computed and saved to disk (as described previously in Das \& Bode 2008). These pixelized shells are used to model the gravitational lensing (\$2.3) and high-redshift SZ distortion ( $\$ 2.4)$ of the microwave background.

For lower redshifts $z<3$, the positions and velocities of all particles in the light cone are also saved to disk for postprocessing. The approximately 55 billion particles thus saved occupy $1.3 \mathrm{~TB}$ of disk space and are used to model the SZ effect (\$2.4) in detail. A friends-of-friends (FoF) halo finder, with a standard linking length $b_{\mathrm{FoF}}$ equal to $1 / 5$ of the mean interparticle spacing, is used to identify dark matter halos and tag the particles belonging to them. This halo catalog is used to model infrared $(\$ 2.5)$ and radio $(\$ 2.6)$ point sources.

As shown in Fig. 11 the halo mass functions measured from particles in the light cone agree well with the semi-analytic fitting formula from Jenkins et al. (2001) for a linking length $b_{\mathrm{FoF}}=0.2$. The number of halos above a minimum mass per unit redshift per square degree is plotted for four min- 
imum masses: $M_{\min }=6.82 \times 10^{12}, 2 \times 10^{13}, 1 \times 10^{14}$, and $3 \times 10^{14} h^{-1} M_{\odot}$. The first limit, the equivalent of 100 particles, is the lowest mass of halos that are populated with point sources. The second value corresponds to massive halos with enough resolution to model the intrahalo gas in greater detail. For any cluster survey, the minimum detectable mass is likely to be a function of redshift, and the last two minimum mass values reflect the range expected for upcoming SZ surveys. The data points in Fig. 1 are measured using all halos in the octant to minimize sample variance, but then are normalized per square degree.

\subsection{Lensing of the Primary $C M B$}

Gravitational lensing is a secondary anisotropy of the microwave background caused by the deflection of primary microwave background photons by intervening large-scalestructure potentials (see Lewis \& Challinor 2006, for a recent review). Lensing produces non-Gaussianities, smooths out features in the power spectrum and moves power from large to small scales. High resolution microwave background experiments, such as ACT, SPT, and Planck, will probe the scales corresponding to the multipole range $500 \lesssim \ell \lesssim 10000$ with unprecedented precision. It will be necessary to include the effect of lensing for performing precision cosmological tests with such datasets. By studying the lensing distortions in the microwave sky, we should also be able to reconstruct the projected matter distribution or the deflection field in the Universe ( $\mathrm{Hu} \&$ Okamoto 2002; Okamoto \& Hu 2003; Hirata \& Seljak 2003; Yoo \& Zaldarriaga 2008). Such reconstruction will help break parameter degeneracies in the microwave background, making it sensitive to dark energy dynamics and neutrino mass (Smith et al. 2008; de Putter et al. 2009). Also, the cross-correlation of microwave background lensing with various tracers of large scale structure will lead to better understanding of galaxy environments, structure formation, geometry and gravity on large scales (Acquaviva et al. 2008; Das \& Spergel 2009; Vallinotto et al. 2009). A realistic simulation of microwave lensing internally consistent with (i.e. based on the same large-scale-structure simulation as) the other simulated secondary components, such as the thermal and kinetic SZ effects and infrared and radio point sources, is essential for training the power spectrum and parameter estimation pipeline. Such simulations are also necessary ingredients in developing realistic lens reconstruction algorithms (Amblard et al. 2004; Perotto et al. 2009). Here we present the salient aspects of a full-sky microwave background lensing simulation at arcminute resolution.

A simulation of the lensed microwave background essentially consists of remapping the points of the primary microwave background sky according to deflection angles derived from a simulated convergence field. Several approaches have been proposed for implementing the remapping as well as for generating the deflection field (Lewis 2005; Das \& Bode 2008; Carbone et al. 2007, 2008; Basak et al. 2008). In this work, we follow Das \& Bode (2008) in all respects, except that we create a full-sky convergence map (instead of the polar cap geometry adopted in that paper) and lens a full-sky primary microwave background map. The details of the algorithm can be found in Das \& Bode (2008) here we delineate the main steps:

1. As described in $\$ 2.2$, at each time step of the largescale-structure simulation, the positions of the particles in a thin shell (of width corresponding to the time step)

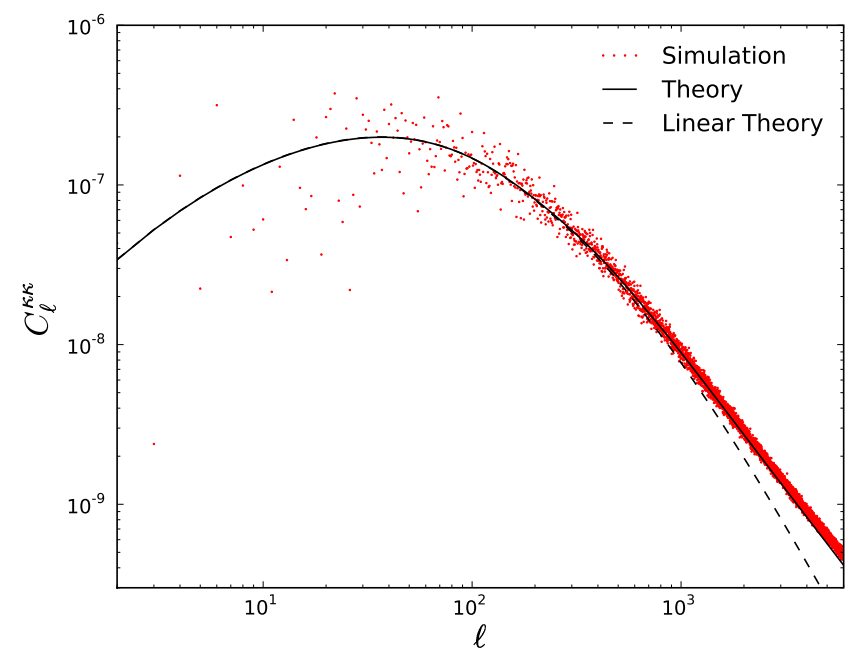

FIG. 2.- Power spectrum of the simulated effective kappa map (dots) compared to theory, with (solid line) and without (dashed line) nonlinear corrections to the matter power spectrum.

are recorded onto the octant of a HEALPix pixelated sphere. Knowing the particle mass and the number of particles falling into each pixel, we generate a surface mass density plane (expressed in mass per steradian) by dividing the total mass in a pixel by the solid angle subtended by it. We then subtract the cosmological mean of the surface density to generate a surface overdensity on each shell.

2. We then generate the effective convergence map $(\kappa)$ up to the maximum redshift in the large-scale-structure simulation $(z=10)$ by combining the surface overdensities in the octant shells with proper geometrical weighting (see, eqns. 15 and 20 in Das \& Bode 2008). To accurately lens the microwave background, we need to include the contribution to the convergence from redshifts beyond the farthest simulated shell $(z>10)$. We do this by adding in a Gaussian random realization for this extra convergence based on a theoretical convergence power spectrum (calculating the contribution to the convergence power from $z>10$ structure). At the end of this process, we have a realization of the effective convergence $\kappa(\hat{n})$ on the octant.

3. We replicate the octant to create a full-sky convergence map. The detailed mapping of the octant to the full-sky is described in $\$ 3$.

4. We invert the relation, $\kappa=\frac{1}{2} \nabla^{2} \phi$, between the convergence and the effective lensing potential $\phi$ in harmonic space to obtain the harmonic components of $\phi$, and generate the deflection field, $\boldsymbol{\alpha}(\hat{n})$, using the relation $\boldsymbol{\alpha}=-\nabla \phi$.

5. We use the deflection field to find the source-plane positions, $\boldsymbol{\theta}_{s}$, corresponding to the observed positions, $\boldsymbol{\theta}$, of the rays (centers of pixels) according to: $\boldsymbol{\theta}_{s}=\boldsymbol{\theta}+\boldsymbol{\alpha}$.

6. Finally, we sample the unlensed microwave background at the source-plane positions using an accurate interpolation technique to produce the lensed microwave background map. 

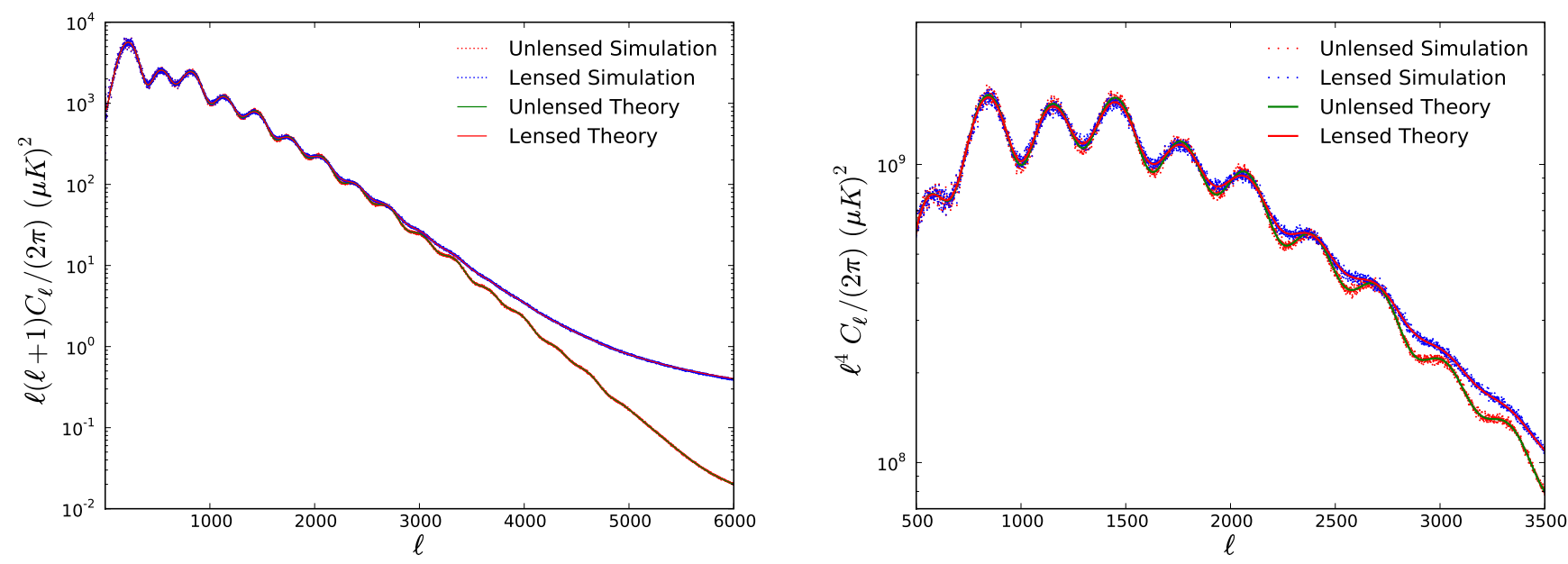

FIG. 3.- Left: Lensed and unlensed microwave background power spectra obtained from the simulation compared with the theoretical models. The blue dots represent the power spectrum obtained from the simulated lensed microwave background map while the red solid line represents the theoretical lensed microwave background power spectrum from CAMB. The red dots represent the power spectrum of the input unlensed map and the green solid line is the theoretical input power spectrum. Note that the simulation and theory lines coincide to high precision. Right: A smaller section of the spectra on the left. Note that the spectrum is multiplied by the fourth power of the multipole to make clearer the difference between lensed and unlensed spectra.

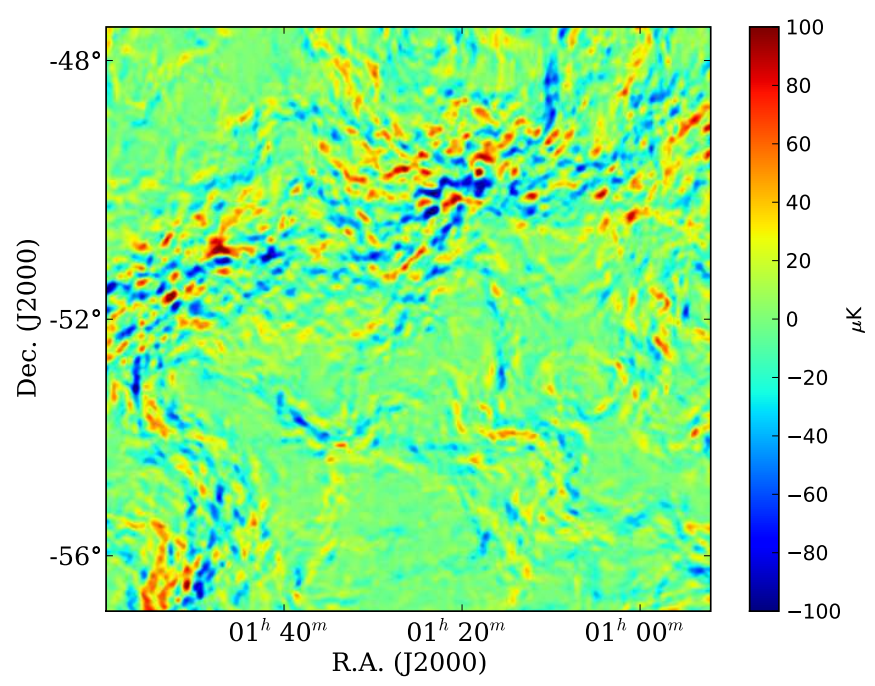

FIG. 4.- Difference between the lensed and the unlensed primary microwave background map in a $\sim 10$ degree $\times 10$ degree patch.

For the results presented here, we have performed the above steps at the HEALPix resolution $N_{\text {side }}=4096$. Then we convert to a map with HEALPix parameter $N_{\text {side }}=8192$ using the alter_alm subroutine included in the HEALPix distribution (Górski et al. 2005).

Fig. 2 compares the power spectrum of the simulated effective $\kappa$ map with two theoretical models: one with and the other without nonlinear corrections (following Smith et al. 2003 ) to the matter power spectrum. The theoretical spectra have been computed with the publicly available $\mathrm{CAMB}^{11}$ code. As expected, the simulated power spectrum agrees better with the theory curve with nonlinear corrections. There are two things worth noticing in the plot: the large scatter at

\footnotetext{
11 www.camb.info
}

the low multipole end of the power spectrum, and the excess power in the simulation beyond $\ell \sim 2000$. The scatter is expected because we generated the full-sky convergence map by replicating an octant. The apparent high multipole excess is a consequence partly of the nonlinear corrections to the theory slightly underpredicting the nonlinear power, and partly of the shot noise due to the discrete nature of the particles.

We computed the power spectrum of the lensed microwave background map and compared it with theoretical expectations. Fig. 3 shows the excellent agreement between the theoretical power spectrum from CAMB and the power spectrum generated from the simulated lensed map. For completeness, we also show the power spectrum of the unlensed input map and the theoretical power spectrum used to generate it. The simulation confirms the theoretical prediction that lensing smoothes out acoustic features in the microwave background and moves power from large to small scales. Parenthetically, we would like to remark that the theoretical power spectra in Figs. 2] \& 3 were generated with CAMB using the parameter k_eta_max_scalar set to $20 \times 1$ I_max_scalar. For k_eta_max_scalar $=2 \times 1$ _max_scalar (which is normally used) the $\kappa$ power spectrum, as well as the lensed microwave background power spectrum, become inaccurate at high multipoles; the $\kappa$ power spectrum is suppressed beyond $\ell \sim 1000$, and the lensed microwave background power spectrum beyond $\ell \sim 4000$.

A particularly interesting quantity is the difference between the lensed and the unlensed microwave background maps. Fig. 4 shows this quantity on a 7 degree $\times 9$ degree patch projected onto a cylindrical-equal-area grid from the HEALPix sphere. The figure shows the striking largescale non-Gaussian features that lensing induces on the microwave sky. Lensing reconstruction techniques use these large-angular-scale correlations of small-scale features in the microwave background to recover the lensing potential.

\subsection{SZ Signal}

The SZ effect is a secondary distortion of the microwave background that results when cosmic microwave radiation 
scatters against free electrons. It is commonly considered to have two main components (Sunyaev \& Zeldovich 1970, 1972). The thermal SZ (TSZ) term arises from inverse Compton scattering of the CMB with hot electrons, predominantly associated with shockheated gas in galaxy clusters and groups. The kinetic SZ (KSZ) term is a Doppler term coming from scattering with electrons having fast peculiar motions. Another common distinction is that the KSZ effect has a nonlinear component associated with the small-scale intracluster medium and a more linear component coming from the large-scale intergalactic medium (IGM). The signal arising from the latter was first calculated for the linear regime by Ostriker \& Vishniac (1986) and Vishniac (1987) and is often referred to as the OV effect. Below, we first write down the formalism for the nonrelativistic limit and then the more general relativistic case.

Modeling the SZ effect requires knowing the number density $n_{e}$, temperature $T_{e}$, and velocity $v_{e}$ of the electron distribution. In the nonrelativistic limit, the change in the CMB temperature at frequency $\nu$ in the direction $\hat{n}$ on the sky is given by

$$
\frac{\Delta T}{T_{\mathrm{CMB}}}(\hat{n})=\left(\frac{\Delta T}{T_{\mathrm{CMB}}}\right)_{\mathrm{tsz}}+\left(\frac{\Delta T}{T_{\mathrm{CMB}}}\right)_{\mathrm{ksz}}=f_{\nu} y-b,
$$

where the dimensionless Compton $y$ and Doppler $b$ parameters,

$$
\begin{aligned}
& y \equiv \frac{k_{B} \sigma_{T}}{m_{e} c^{2}} \int n_{e} T_{e} \mathrm{~d} l=\int \theta_{e} \mathrm{~d} \tau, \\
& b \equiv \frac{\sigma_{\mathrm{T}}}{c} \int n_{e} v_{\mathrm{los}} \mathrm{d} l=\int \beta_{\mathrm{los}} \mathrm{d} \tau,
\end{aligned}
$$

are proportional to integrals of the electron pressure and momentum along the line of sight (los), respectively. The dimensionless temperature, line-of-sight peculiar velocity, and the optical depth through a path length $\mathrm{d} l$ are given by

$$
\begin{gathered}
\theta_{e} \equiv \frac{k_{\mathrm{B}} T_{e}}{m_{e} c^{2}}=1.96 \times 10^{-3}\left(\frac{T_{e}}{\mathrm{keV}}\right), \\
\beta_{\mathrm{los}} \equiv \frac{v_{\mathrm{los}}}{c}=3.34 \times 10^{-4}\left(\frac{v_{\mathrm{los}}}{100 \mathrm{~km} / \mathrm{s}}\right), \\
\mathrm{d} \tau \equiv \sigma_{\mathrm{T}} n_{e} \mathrm{~d} l=2.05 \times 10^{-3}\left(\frac{n_{e}}{10^{-3} \mathrm{~cm}^{3}}\right)\left(\frac{\mathrm{d} l}{\mathrm{Mpc}}\right),
\end{gathered}
$$

respectively. For a typical cluster, the Compton $y$ parameter is expected to be approximately an order of magnitude larger than the Doppler $b$ parameter.

The nonrelativistic TSZ component has a frequency dependence as specified by the function,

$$
f_{\nu} \equiv x \operatorname{coth}(x / 2)-4, \quad x \equiv h \nu /\left(k_{\mathrm{B}} T_{\mathrm{CMB}}\right),
$$

which has a null at $\nu \approx 218 \mathrm{GHz}$. The distortion appears as a temperature decrement at lower frequencies and as an increment at higher frequencies relative to the null. The nonrelativistic KSZ component is independent of frequency, but the sign of the distortion depends on the sign of the line-ofsight velocity. We chose the convention where $v_{\text {los }}>0$ if the electrons are moving away from the observer.

In the general, relativistic case, the change in the CMB tem-

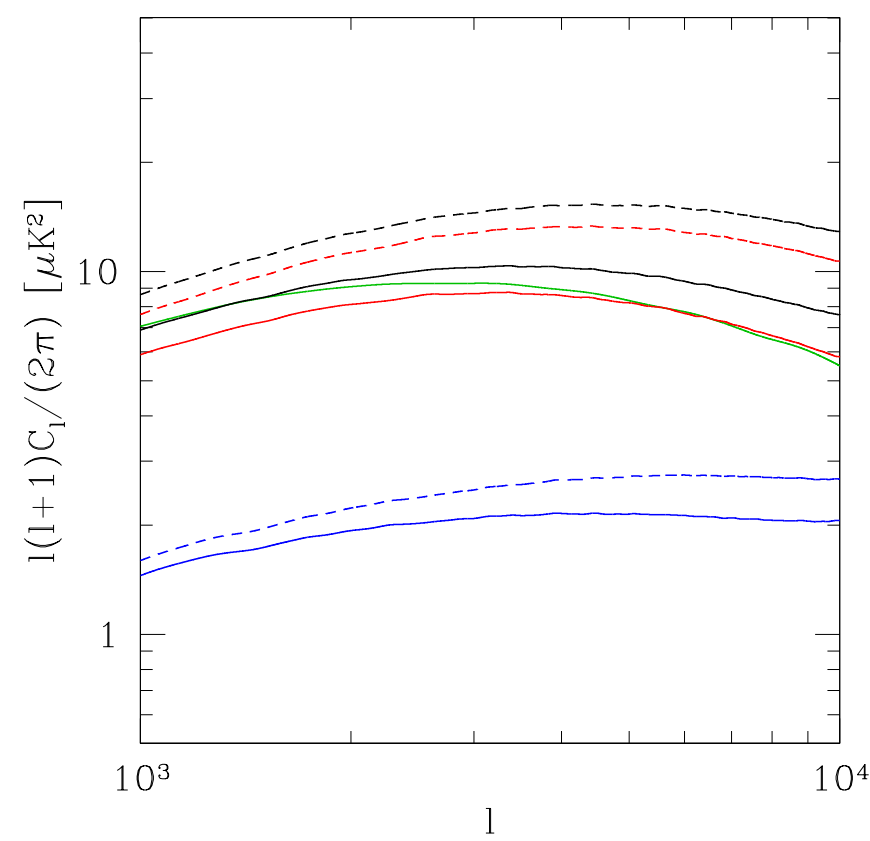

FIG. 5.- Power spectra of the Sunyaev-Zel'dovich signals at 148 GHz. Shown are the power spectra of the thermal SZ component (red), the kinetic SZ component (blue), and the full SZ signal (black). The solid lines represent the model in this simulation, and the dashed lines represent a model with no feedback or star formation (adiabatic model Bode et al. 2009; Trac et al. 2009). The green line shows the comparison with the thermal SZ power spectrum derived analytically from Komatsu \& Seliak (2002) and taken from the WMAP LAMBDA website (http://lambda.gsfc.nasa.gov/product/map/current/).

perature at frequency $\nu$ in the direction $\hat{n}$ is given by

$$
\begin{gathered}
\frac{\Delta T}{T_{\mathrm{CMB}}}(\hat{n})=\int\left[\theta_{e}\left(Y_{0}+\theta_{e} Y_{1}+\theta_{e}^{2} Y_{2}+\theta_{e}^{3} Y_{3}+\theta_{e}^{4} Y_{4}\right)\right. \\
+\beta^{2}\left[\frac{1}{3} Y_{0}+\theta_{e}\left(\frac{5}{6} Y_{0}+\frac{2}{3} Y_{1}\right)\right] \\
\left.-\beta_{\mathrm{los}}\left(1+\theta_{e} C_{1}+\theta_{e}^{2} C_{2}\right)\right] \mathrm{d} \tau,
\end{gathered}
$$

where the $Y$ 's and $C$ 's are known frequency-dependent coefficients (Nozawa et al. 1998). Note that Eq. 1 contains only the first-order terms in Eq. 8, and that $f_{\nu}=Y_{0}$. We use Eq. 8 to calculate the full SZ signal in these simulations.

Sky maps of the SZ effect are made by tracing through the simulated electron distribution and projecting the accumulated temperature fluctuations onto a HEALPix grid with Nside $=8192$. Using the saved simulation data described in $\$ 2.2$ SZ maps are constructed with contributions from 3 components: massive halos at $z<3$, low-mass halos and the intergalactic medium at $z<3$, and the high-redshift Universe for $3 \leq z<10$.

\subsubsection{Gas model for massive halos at $z<3$}

The hot gas distribution associated with massive dark matter halos is calculated using a hydrostatic equilibrium model (Ostriker et al. 2005; Bode et al. 2007, 2009). Here we summarize the general method and refer the reader to Bode et al. (2009) for additional details, in particular on calibrating star formation and feedback against recent optical and X-ray observations. In the large-scale-structure simulation, massive 
dark matter halos with $M_{\mathrm{FoF}}>2 \times 10^{13} h^{-1} M_{\odot}$ and $z<3$ are postprocessed to include intrahalo gas and stars. These halos are expected to be the dominant contribution to the TSZ terms in Eqs. 1 and 8 for the scales of interest $(1000 \lesssim l \lesssim 10000)$. Lower mass halos are accounted for differently, as we explain later.

For each massive halo, the collection of N-body particles tagged by the FoF finder are enclosed in a nonperiodic, Cartesian mesh that is centered on the center of mass, has one axis parallel to the line of sight, and a cell-spacing that is twice the $\mathrm{N}$-body spline softening length. The particles are assigned using a cloud-in-cell scheme to define the matter density $\rho_{m}$ on the mesh. The gravitational potential $\phi$ is then calculated using a nonperiodic Fast Fourier Transform (FFT), and the location of the potential minimum is chosen as the halo center. This procedure preserves the concentration, substructure, and triaxiality of the dark matter halo.

Assuming hydrostatic equilibrium and a polytropic equation of state, the corresponding gas density $\rho_{g}$ and pressure $P_{g}$ on the same mesh are given by

$$
\begin{aligned}
& \rho_{g}=\rho_{g, 0} \psi^{1 /(\Gamma-1)}, \\
& P_{g}=P_{g, 0} \psi^{\Gamma /(\Gamma-1)},
\end{aligned}
$$

respectively, where the dimensionless temperature variable,

$$
\psi \equiv 1-\left(\frac{\Gamma-1}{\Gamma}\right)\left(\frac{\rho_{g, 0}}{P_{g, 0}}\right)\left(\phi-\phi_{0}\right),
$$

is related to the gravitational potential. Bode et al. (2009) adopted a polytropic index $\Gamma=1.2$ (consistent with the value of $\Gamma$ adopted by other workers and the value found in hydrodynamic simulations) and determined the other two free parameters for the polytropic model, namely the central gas density $\rho_{g, 0}$ and pressure $P_{g, 0}$, by matching the gas mass fraction and temperature to recent X-ray observations (Vikhlinin et al. 2006; Sun et al. 2009). The electrons are assumed to have the same distribution of temperatures and velocities as the fully ionized gas.

For calculating the KSZ terms in Eq. 11 and 8, the gas velocity on the mesh can be modeled in two ways. The velocity field can be taken from the dark matter particles; this approach allows for velocity substructure, although the dispersion would be inconsistent with the hydrostatic equilibrium assumption. Alternatively, the entire gas distribution can be assigned a single, mass-weighted average peculiar velocity; this is the approach we adopt for self-consistency. Comparing the two options, we underestimate the temperature variance $<\Delta T_{\mathrm{ksz}}^{2}>$ from massive halos alone by only $\sim 4 \%$ when ignoring velocity substructure. Correspondingly, the KSZ angular power spectrum from massive halos alone is also underestimated, but only by $<1 \%$ at $l=1000$ and $\sim 6 \%$ at $l=10000$.

When a given halo is added to the SZ maps, mass and momentum are conserved by requiring that the baryonic mass be equal to the cosmic baryon fraction $\Omega_{\mathrm{b}} / \Omega_{\mathrm{m}}$ times the halo mass $M_{\mathrm{FoF}}$. This is accomplished by first sorting the mesh cells by their gravitational potential, from most negative to least negative. The mesh is then traversed in order and cells are tagged until the accumulated baryonic mass, in gas and stars, reaches the mass limit. The outer radius of the gas is usually beyond the cluster virial radius. Remaining cells do not contribute any SZ signal. This procedure results in a triaxial halo gas distribution with a physically-motivated cutoff.

We find that the gas associated with these massive halos

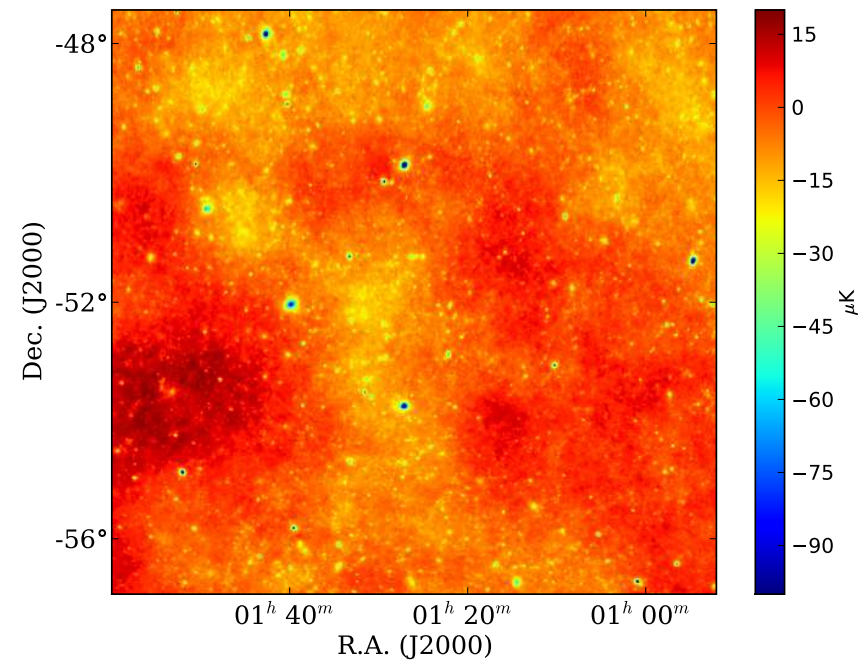

FIG. 6.- The full Sunyaev-Zel'dovich signal at $148 \mathrm{GHz}$, including thermal and kinetic SZ contributions from both the galaxy clusters and the intergalactic medium, in a $\sim 10$ degree $\times 10$ degree patch.

makes up approximately half of the TSZ flux, but it is the dominant contribution to the TSZ angular power spectrum. It accounts for $\sim 90 \%$ of the total TSZ power for the scales of interest $(1000 \lesssim l \lesssim 10000)$. On the contrary, this gas accounts for only $\sim 15 \%$ of the total KSZ power because of the relative importance of the IGM to this signal, as discussed in $\$ 2.4 .2$ and $\$ 2.4 .3$

\subsubsection{Low-mass halos and intergalactic medium at $z<3$}

Low-mass halos and the intergalactic medium at $z<3$ are modeled using all other saved N-body particles not associated with the massive dark matter halos discussed in $\$ 2.4 .1$ While we are able to identify halos down to $M_{\mathrm{FoF}}=6.82 \times$ $10^{12} h^{-1} M_{\odot}$ (100 particles), there is insufficient resolution to accurately apply the hydrostatic equilibrium model. Instead, an alternative approach motivated by the virial theorem is adopted, one that can also be extended to particles not associated with identified halos. The intergalactic medium is expected to contribute only a small fraction of the TSZ signal, but it is very important to include it for calculating the KSZ signal.

According to the virial theorem, the internal energy of a virialized halo is twice its gravitational potential energy. During the formation of a halo, the dark matter undergoes violent relaxation and the gas gets shock-heated. The gas temperature can be estimated directly from the halo mass and used to model the TSZ signal (e.g. Cohn \& White 2009), but we chose to calculate the gas temperature from the velocity dispersion of the dark matter. This approach is then applicable to all particles rather than just ones in identified halos.

For each particle, the local density $\rho_{m}$ and velocity dispersion $\sigma_{v}$ are calculated using 32 neighboring particles. For calculating the SZ terms, the effective pressure and momentum of the gas is taken as

$$
\begin{gathered}
P_{g}=\frac{\Omega_{\mathrm{b}}}{\Omega_{\mathrm{m}}}\left[\frac{(\gamma-1) \rho_{m} \sigma_{v}^{2}}{2 \mu m_{\mathrm{H}} k_{\mathrm{B}}}\right], \\
(\rho v)_{g}=\frac{\Omega_{\mathrm{b}}}{\Omega_{\mathrm{m}}}(\rho v)_{m},
\end{gathered}
$$


where $\gamma=5 / 3$ is the ideal gas adiabatic index and $\mu=4 /(3+$ $\left.5 X_{\mathrm{H}}\right)=0.588$ is the mean atomic weight for a fully ionized gas. Helium reionization is assumed to occur instantaneously at $z=3$. A temperature floor of $10^{4} \mathrm{~K}$ is imposed for the photoionized intergalactic medium.

When tracing through a given particle and projecting onto a HEALPix grid, the effective solid angle subtended by the particle is taken into account. A nearby, low-density particle can span a number of map pixels and simply assigning it to a single pixel will result in a large and incorrect temperature fluctuation. Mass and momentum are also conserved. Since the entire gas distribution at $z<3$ is accounted for, the maps have a smooth transition from the concentrated halos to the diffuse intergalactic medium.

We find that the low-mass halos and the IGM make up approximately half of the TSZ flux, but contribute to the angular power spectrum only by $\sim 10 \%$ at $l=1000$ and $\sim 15 \%$ at $l=$ 10000. This is in agreement with Hallman et al. (2007) who used a high-resolution hydrodynamic simulation and found that while roughly one-third of the TSZ flux comes from lowmass halos $\left(M<5 \times 10^{13} M_{\odot}\right)$ and the IGM, these components add $\lesssim 10 \%$ to the TSZ angular power spectrum (for $\sigma_{8}=$ 0.9). Hallman et al. (2009) also found that the angular power spectrum of the filamentary warm-hot intergalactic medium (WHIM Cen \& Ostriker 1999; Davé et al. 2001) is only $\lesssim$ $1 \%$ of the total. Furthermore, Hernández-Monteagudo et al. (2006) found that while lower density gas $(\delta \lesssim 10)$ makes up approximately half of the baryon budget, it gives rise to only $\sim 5 \%$ of the TSZ flux in the local universe.

For low-mass halos in the mass range $6.82 \times 10^{12}<$ $M_{\mathrm{FoF}}\left(h^{-1} M_{\odot}\right)<2 \times 10^{13}$, there are adequate particle numbers (100 to 282 ) to estimate the halo velocity dispersion. The TSZ signal from this component is sufficiently well approximated with the above method, considering that these halos contribute $\lesssim 10 \%$ to the TSZ angular power spectrum. However, for filaments with typical overdensities of 10 , there are only 10 particles per comoving $\left(h^{-1} \mathrm{Mpc}\right)^{3}$. While it is difficult to calculate the velocity dispersion accurately, an approximate solution is acceptable since the filaments contribute only $\lesssim 1 \%$ to the angular power spectrum.

The KSZ signal from the low-mass halos and the IGM are relatively more important. We find that at $l=1000$, they account for $\sim 15 \%$ of the total power, comparable to the contribution from massive halos, and at $l=10000$, they account for $\sim 40 \%$. The majority of the KSZ signal is from the highredshift universe $(3<z<10)$, which we discuss in $\$ 2.4 .3$. The $\mathrm{KSZ}$ signal is relatively less sensitive to resolution since the bulk peculiar momentum is well captured in the large-scalestructure simulation. While velocity substructure is not well resolved, we have already demonstrated in $\$ 2.4$.1 that ignoring it only results in percent level differences. However, given the modest resolution of the large-scale-structure simulation, we caution against using the maps for detailed studies of the SZ signal from the WHIM and IGM.

\subsubsection{High-redshift Universe at $3 \leq z<10$}

The high-redshift universe at $3 \leq z<10$ is modeled using the redshift shells containing the surface density fields for mass $\Sigma_{m}$ and line-of-sight momentum $\Sigma_{m v}$. At these higher redshifts, it is very reasonable to assume that the gas traces the matter distribution because the scales of interest are mostly still in the linear regime. Furthermore, since both the nonlinear mass and the collapsed mass fraction above a fixed mass rapidly decrease towards higher redshifts, the typical temperature of the gas is predominantly set by photoionization rather than shockheating.

For each redshift shell, the electron temperature, line-ofsight peculiar velocity, and column density are taken to be

$$
\begin{gathered}
T_{e}=10^{4} \mathrm{~K}, \\
v_{\mathrm{los}}=\frac{\Sigma_{m v}}{\Sigma_{m}}, \\
N_{e}=\left(\frac{\Omega_{\mathrm{b}}}{\Omega_{\mathrm{m}}}\right)\left(\frac{X_{\mathrm{H}}}{m_{\mathrm{H}}}+\frac{Y_{\mathrm{He}}}{m_{\mathrm{He}}}\right) \Sigma_{m} .
\end{gathered}
$$

Hydrogen reionization is assumed to occur instantaneously at $z=10$ and helium is only singly ionized. The Thomson optical depth for electron scattering is $\tau_{\mathrm{T}} \approx 0.08$, consistent with the WMAP 5-year results (Dunkley et al. 2009). Since the electron distribution is warm rather than hot, the SZ temperature fluctuations are nonrelativistic and Eq. 8 reduces to Eq. 1. There is only a very small contribution to the TSZ signal from the warm electron distribution. The average Compton $y$ parameter is only $\sim 10^{-7}$ over this redshift range. However, there is a substantial contribution to the KSZ signal because of the large, coherent velocities in the IGM.

Since the light cone is constructed by replicating and stacking from the periodic simulation box without random rotations, this procedure introduces excess power in the KSZ signal at $l \lesssim 500$. The excess power shows up in the intergalactic medium component, but not in the halo component previously discussed in $\$ 2.4 .1$ Therefore, the intergalactic medium component of the KSZ, from the full redshift range $0<z<10$, is filtered before adding it to the final SZ maps. A simple filter $w(l)=1-\exp \left[-(l / 500)^{2}\right]$ is chosen to suppress the large-scale excess, but preserve power at $l>1000$. The remaining low- $l$ power is now more consistent with the level expected for the large-scale OV signal.

Since the simple filtering modifies the signal at $l<1000$, the maps should not be used to predict the KSZ signal at these scales. Furthermore, we have neglected to model the contribution from the inhomogeneous reionization epoch. This component is expected to be larger than the contribution from the reionized universe $(z \lesssim 6)$ at $l \lesssim 1000$ (e.g. Santos et al. 2003; McQuinn et al. 2005; Zahn et al. 2005). The KSZ signal on these larger scales is subdominant compared to the lensed microwave background and to the TSZ signal at frequencies away from the null.

\subsection{Infrared Point Sources}

Star formation in the Universe leads to the production of dust grains which absorb the ultraviolet radiation in the environment and re-radiate it in the infrared range. In the context of microwave background observations, the redshifted infrared emission of these sources constitutes an important contaminant on small angular scales at frequencies higher than $\sim 150 \mathrm{GHz}$. In this section, we attempt to model the angular anisotropies that infrared (IR) galaxies introduce in the microwave sky by combining the cosmological simulation presented above with a model for the infrared galaxy population, which is partially based upon Righi et al. (2008).

\subsubsection{The basics of the infrared model}


We first approximate the spectral energy distribution (SED) of the infrared galaxies by a modified black body of the form

$$
L_{\nu} \propto \phi\left(\nu, T_{\text {dust }}\right) \equiv \nu^{\beta}\left[B_{\nu}\left(T_{\text {dust }}\right)-B_{\nu}\left(T_{\text {cmb }}\right)\right],
$$

where $\beta$ is a spectral index, $T_{\mathrm{cmb}}$ is the primary microwave background monopole temperature, and $T_{\text {dust }}$ is the dust temperature evolving in redshift as in Blain (1999):

$$
T_{\text {dust }}(z)=\left[T_{0}^{4+\beta}+T_{\mathrm{cmb}}^{4+\beta}\left((1+z)^{4+\beta}-1\right)\right]^{1 /(4+\beta)} .
$$

Here $T_{0}$ is the dust temperature if the galaxy was at $z=0$, and the symbol $B_{\nu}(T)$ denotes the black body spectral intensity at temperature $T$. As we shall see below, the free model parameters $\beta$ and $T_{0}$ will determine the spectral index for the effective power law describing the change of the infrared intensity versus frequency, $I_{\nu} \propto \nu^{\alpha}$ (i.e., $\alpha=\alpha\left(\beta, T_{0}\right)$ ).

The star formation rate (SFR) in a given galaxy seems to be linearly correlated with the dust luminosity (Kennicutt relation, Kennicutt 1998). At the same time, there is observational evidence that the SFR reached a maximum at epochs corresponding to $z \sim 2-4$ (Hopkins \& Beacom 2006). These two factors combined suggest introducing a redshift window function for the infrared luminosities in galaxies close to the measured SFR behavior. In our model, we adopt the dependence

$$
W_{S F R}(z) \propto \exp (-1 / z) \exp \left(-(z / 3.5)^{2}\right) .
$$

This window however gives more weight to the redsfhift range $z \in[1,2]$ if compared to the data of Hopkins \& Beacom (2006), due mainly to the absence of halos in our simulation at $z>3$.

In practice, given the simulation halo catalog, our approach consists of populating each halo with a number of galaxies with a given infrared luminosity. We adopt a mass range limited by $M_{1}, M_{2}\left(M_{1}<M_{2}\right)$, in such a way that halos below $M_{1}$ will host no infrared galaxies. Halos with masses $M \in$ $\left[M_{1}, M_{2}\right]$ will typically host one infrared galaxy of total infrared luminosity $L_{I R}(M, z)=W_{S F R}(z) \cdot W_{\text {cool }}(M) \cdot L_{\star} \cdot\left(M / M_{2}\right)$. For more massive halos, the average number of infrared galaxies will be given by $M / M_{2}$, each of them with a total infrared luminosity given by $L_{I R}(M, z)=W_{S F R}(z) \cdot W_{\text {cool }}(M) \cdot L_{\star}$. The function $W_{\text {cool }}(M)=\exp \left(-M / M_{\text {cool }}\right)$ accounts for the fact that bigger halos need longer times to cool down before being able to form stars. $L_{\star}$ defines a luminosity parameter. The parameters $M_{1}, M_{2}, L_{\star}$ and $M_{\text {cool }}$, combined with the spectral parameters $\beta$ and $T_{\text {dust }}$ introduced above, will define our infrared model. In the simulations, the actual number of infrared galaxies present in a given halo is driven by a Poissonian realization of its average number. We also introduced an effective scatter of $15 \%$ in the mean adopted value for the dust spectral index and in the infrared luminosity of each galaxy, partially motivated by the scatter in the effective spectral index of the intensity found in the analyses by Knox et al. (2004).

\subsubsection{The angular power spectrum}

Below we calculate the angular power spectrum of our infrared model theoretically. This allows us to approximate the power spectrum efficiently, after adjustments to the model parameters, without generating HEALPix maps. For each IR galaxy, the spectral luminosity $L_{\nu}$ can be computed from the bolometric luminosity and the adopted form of the SED:

$$
L_{\nu}=\frac{L_{I R}(M, z)}{\int d \nu \phi\left(\nu, T_{\text {dust }}\right)} \phi\left(\nu, T_{\text {dust }}\right)
$$

where $T_{\text {dust }}$ is a function of redshift (Eq. 18). For a flat Universe, the measured spectral flux is therefore given by

$$
F_{\nu}=\frac{L_{\nu^{\prime}}}{4 \pi r^{2}(1+z)},
$$

where $\nu=\nu^{\prime} /(1+z)$ and $r$ is the comoving distance to the IR galaxy placed at redshift $z(r)$. The average spectral intensity generated by the IR galaxy population can then be written as the sum of the contribution of all galaxies per unit solid angle:

$$
\bar{I}_{\nu}=\int d r r^{2} \int d M \frac{d n}{d M}(r) N_{g}(M) \frac{a(r) L_{\nu^{\prime}}(M, r)}{4 \pi r^{2}}
$$

The comoving halo mass function is given by $d n / d M$, and $N_{g}(M)$ provides the average number of infrared galaxies in a halo of mass $M$. The cosmological scale factor is expressed by $a(r)$, and the typical spectral luminosity for each of the infrared galaxies in the halo is given by $L_{\nu^{\prime}}(M, r)$, with $\nu^{\prime}$ being the frequency observed at redshift $z(r) . L_{\nu^{\prime}}(M, r)$ is determined by the model described in $\$ 2.5 .1$ and Eq. 20 .

However, we are interested in the angular fluctuations of the infrared spectral intensity, and this is linked to the angular variation of the number of infrared sources. The number of infrared galaxies is driven a priori by Poisson statistics. However, halos tend to cluster in overdense regions of the large scale matter density field, and this introduces another modulation in the infrared galaxy number density that is known as the correlation term (Haiman \& Knox 2000; Song et al. 2003; Righi et al. 2008). In terms of the spectral intensity $I_{\nu}$, it is easy to show that the Poisson term for the angular anisotropies generated by the infrared galaxy population can be written as (Righi et al. 2008)

$$
C_{l}^{P}=\int d r r^{2} \int d M \frac{d n}{d M}(r) N_{g}(M)\left(\frac{a(r) L_{\nu^{\prime}}(M, r)}{4 \pi r^{2}}\right)^{2},
$$

whereas the correlation or clustering term depends upon the halo power spectrum:

$C_{l}^{C}=\int \frac{d r}{r^{2}} P_{m}(k, r)\left[\int d M \frac{d n}{d M}(r) b(M, r) N_{g}(M) \frac{a(r) L_{\nu^{\prime}}(M, r)}{4 \pi}\right]^{2}$

where $k=(l+1 / 2) / r$. The bias factor $b(M, r)$ expresses how halos are distributed compared to dark matter, in such a way that $b^{2}(M, r)$ is defined as the ratio of the halo power spectrum and the matter power spectrum $\left(P_{m}(k, r)\right)$. Here we have adopted the model by Sheth \& Tormen (1999), which provides a good fit (few percent accuracy) to the simulated halo power spectrum. The last two equations are obtained after using the Limber approximation, which should be accurate to better than $2 \%$ for $l>20$ (e.g., Verde et al. 2000).

\subsubsection{Observational constraints}

The parameter space is swept in such a way that our infrared model satisfies the following observational constraints:

- The Cosmic Infrared Background (CIB), as estimated in Fixsen et al. (1998) from COBE/FIRAS, is the upper limit for all total infrared intensity that our model provides. 

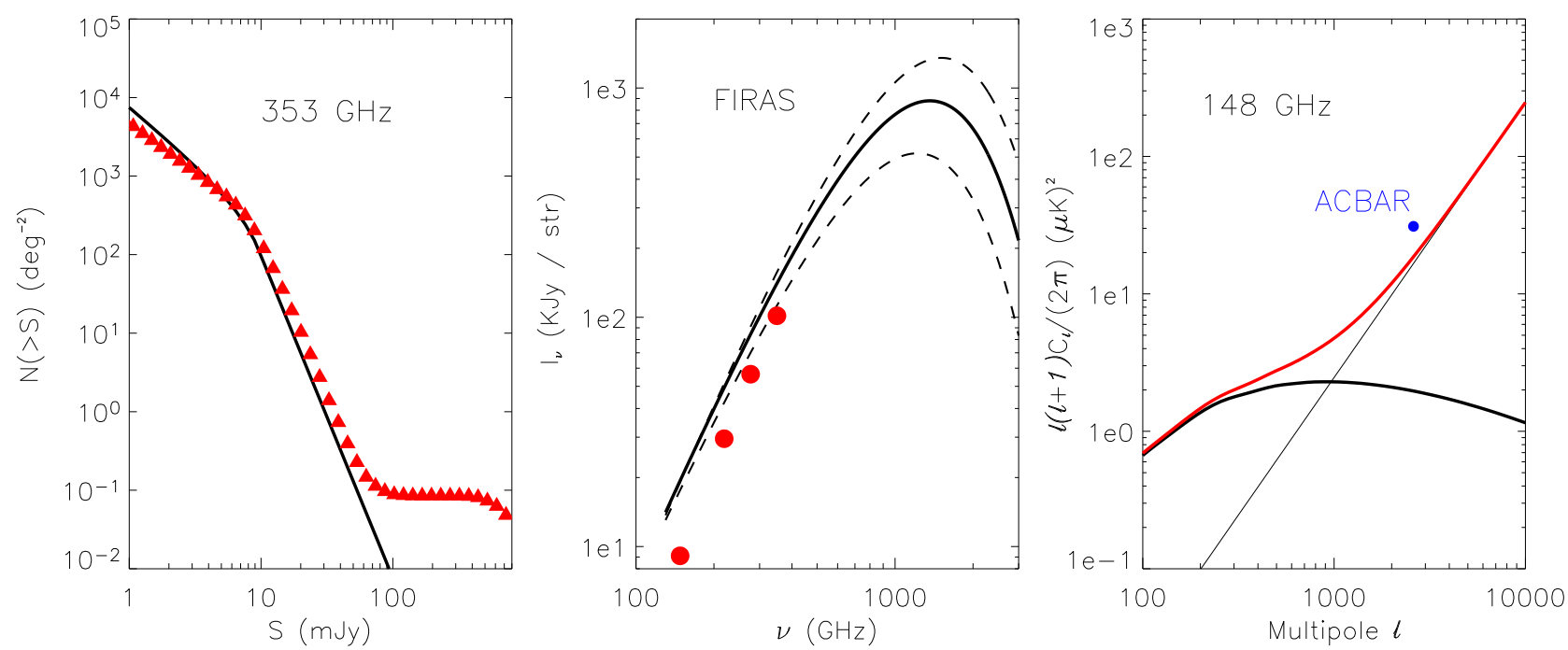

FIG. 7.-Left panel: Cumulative source counts of our infrared model at $353 \mathrm{GHz}$ versus flux (red triangles). The solid line corresponds to the fit to the SCUBA data at the same frequency (Coppin et al. 2006). The high flux sources match the observed BLAST counts (Patanchon et al. 2009 and BLAST team, private communication). Middle panel: Contribution of our infrared source population to the total cosmic infrared background observed by COBE/FIRAS (Fixsen et al. 1998). We show the three observing frequencies of ACT plus $353 \mathrm{GHz}$. Dashed lines indicate $68 \%$ confidence regions. Right panel: Theoretical estimate of the angular power spectrum of our mock infrared source population. The thick black solid line corresponds to the clustering term, and the thin line to the Poisson term. The total is given by the red line, which is below the upper limit suggested by ACBAR at $l=2600$ (Reichardt et al. 2009).

- The choice for spectral parameters $\beta$ and $T_{\text {dust }}$ must be such that the effective spectral index for the spectral intensity $\left(I_{\nu} \propto \nu^{\alpha}\right)$ is close to $\alpha \approx 2.6$ in the range $\nu \in[145,350] \mathrm{GHz}$. This value is found in Knox et al. (2004), and is based on the projection to high redshifts of the analyses of Dunne et al. (2000) of a low redshift infrared galaxy sample. Nearby galaxies show harder spectral indexes, while high $z$ sources are closer to the peak of the dust SED and hence provide a shallower slope. The quoted value of the spectral index is practically equivalent to the slope of the CIB at the same frequency range. Thus, imposing $\alpha \approx 2.6$ makes the infrared background generated by our infrared sources have a slope close to that of the CIB in the frequency range $\nu \in[145,350] \mathrm{GHz}$.

- The source counts of our infrared model must be compatible to the source counts provided by SCUBA (Coppin et al. 2006) in the flux range $S_{353 \mathrm{GHz}} \in[1,50]$ $\mathrm{mJy}$. These source counts are in agreement with a number of other submillimeter observations (e.g. Austermann et al. 2009).

- The source counts of our infrared model must be compatible with the high flux infrared source population recently seen by BLAST (Patanchon et al. 2009 and BLAST team, private communication).

- The clustered component of the the angular power spectrum should dominate over the Poisson term for $l<$ 1000 as observed by Spitzer (Lagache et al. 2007) and BLAST (Viero et al. 2009).

- The angular power spectrum introduced by our IR source population should not exceed the upper limit derived from ACBAR observations at $150 \mathrm{GHz}$ after fixing $\sigma_{8}$ to the WMAP 5-year best-fit value with a running spectral index $\left(\sigma_{8}=0.81\right)$, i.e. $l^{2} C_{l} /(2 \pi)<$ $31(\mu K)^{2}$ at $l=2600$ (Reichardt et al. 2009).

\subsubsection{Final model}

We modeled two different source populations: the basic population essentially defines the properties of the whole IR model, whereas the very high flux population matches the high flux IR source counts detected by BLAST (BLAST team, private communication). Each of these two models requires a different choice for the parameter set, as shown in Table 1.

In order to avoid the limitation imposed by the minimum mass of the halo resolved in our simulation, we added a mock catalog of halos of masses between $10^{9} M_{\odot}$ and the minimum resolved halo mass, $M_{\min }=6.82 \times 10^{12} h^{-1} M_{\odot}=$ $9.61 \times 10^{12} M_{\odot}$. In order to do this, we assumed that the mass function in this mass range was dictated by the fit of Jenkins et al. (2001). We used the population of low mass halos resolved in the simulation as tracers of the mock halos appended in this low mass range. We divided the mass range $\left[10^{9} M_{\odot}, M_{\min }\right]$ in logarithmically spaced bins, and, in each of these bins, computed the average number of halos per resolved halo that is found in a thin mass interval centered at $M_{c}=1.10 \times 10^{13} M_{\odot}$. That is, for each resolved halo of mass close to $M_{c}$, we computed the average number of halos present in each of those low mass bins, and subsequently made a Poissonian realization of this average. The masses of these new mock halos are randomly distributed uniformly within the corresponding mass bin width. These mock halos were randomly distributed (with a Gaussian profile) in spheres of roughly 100 times the parent (resolved) halo virial radius, which should correspond to $\sim 10 \mathrm{Mpc}$. Therefore, for each real halo of mass close to $M_{c}$, we populated a cloud of lower mass halos in an sphere of roughly $10 \mathrm{Mpc}$ radius and centered on the parent halo itself. This procedure provides our simulation with a low mass halo catalog that is not Poisson distributed, but rather is correlated to the population of small but resolved halos close to $M=M_{c}$. In practice, only mock halos of mass above $M_{1}$ were generated, since they are the 
TABLE 1

PARAMETERS FOR THE TWO POPULATIONS CONSIDERED FOR THE INFRARED POINT SOURCE MODEL

\begin{tabular}{ccccccc}
\hline \hline Model & $\beta$ & $\begin{array}{c}T_{0} \\
(K)\end{array}$ & $\begin{array}{c}M_{1} \\
\left(M_{\odot}\right)\end{array}$ & $\begin{array}{c}M_{2} \\
\left(M_{\odot}\right)\end{array}$ & $\begin{array}{c}M_{\text {cool }} \\
\left(M_{\odot}\right)\end{array}$ & $\begin{array}{c}L_{\star} \\
\left(L_{\odot}\right)\end{array}$ \\
\hline Basic & 1.4 & 25 & $2.5 \times 10^{11}$ & $3 \times 10^{13}$ & $5 \times 10^{14}$ & $1.25 \times 10^{12}$ \\
Very High Flux & 1.3 & 40 & $5 \times 10^{14}$ & $1 \times 10^{15}$ & $8 \times 10^{14}$ & $6 \times 10^{14}$ \\
\hline
\end{tabular}

ones eventually hosting IR galaxies.

The very high flux population consists of few $(\sim 300$ in the whole octant) infrared galaxies above $200 \mathrm{mJy}$ at $353 \mathrm{GHz}$. This very high flux population models the tail observed by BLAST at 250, 350 and $500 \mu \mathrm{m}$ (BLAST team, private communication). The high flux tail should be at or above the 10 mJy level in the $145-220 \mathrm{GHz}$ channels, contributing significantly to the power spectrum. Some of this very high flux population includes sources believed to be lensed and magnified by the intervening structure (Lima et al. 2009).

The total cumulative source counts above a given flux threshold for the sum of the basic and the very high flux populations are displayed in the left panel of Fig. 7] We show fluxes above $1 \mathrm{mJy}$ (which is about SCUBA's detection threshold), although our IR source population extends to dimmer fluxes. It is actually the numerous but dim IR source population that is responsible for most of the CIB. In our model, our IR source population generates between $50 \%$ and $80 \%$ of the total CIB detected by FIRAS (middle panel). This is consistent with BLAST observations (Marsden et al. 2009; Devlin et al. 2009), which indicate that most, and perhaps all, of the CIB is generated by infrared sources.

In the right panel, we display the expected angular power spectrum of our total IR source population. At very high multipoles the Poisson term must dominate, whereas the clustering term takes over at $l \sim 1,000$. Again due to the construction of the light cone by replicating and stacking the periodic $\mathrm{N}$-body simulation box without random rotations, excess power is introduced in the infrared point source signal at $l \lesssim 300$, as was the case for the kSZ signal from the intergalactic medium (see $\S 2.4 .3$. We do not filter this signal to suppress the low- $l$ excess as we did for the $\mathrm{kSZ}$, as this signal consists of discrete sources instead of a smooth component, and such a filter would disturb the real space point source distribution. For $l>300$, the theoretical expectation of the angular power spectrum coincides with the actual power spectrum from the simulated infrared map.

The particular values for the model parameters for each of the two populations are displayed in Table 1. Note that the masses in this table refer to friends-of-friends halo masses. For the basic population, the values of the dust temperature and infrared luminosity are not far from those found by Dye et al. (2009) when comparing BLAST infrared sources to radio and mid-infrared counterparts in the Chandra Deep Field South. For the few galaxies in the very high flux population, characteristic masses and infrared luminosities must be increased by roughly an order of magnitude, whereas the typical dust temperature increases from 25 to $40 \mathrm{~K}$.

\subsection{Radio Point Sources}

Our model for radio point sources follows in spirit the treatment of the subject by Wilman et al. (2008, hereafter W08). Similar to W08,we separate the radio-loud active galactic nuclei (AGNs) into two populations, a high-power family that evolves strongly with redshift, and a low-power one with mild redshift evolution. These two populations can be roughly identified with the type II and I classification of radio sources by Fanaroff \& Riley (1974), respectively (however, see discussions in Willott et al. 2001). We also follow W08 and Jackson \& Wall (1999) to employ a relativistic beaming scheme to separate the contributions to the total flux from the compact core and the extended lobes of radio sources.

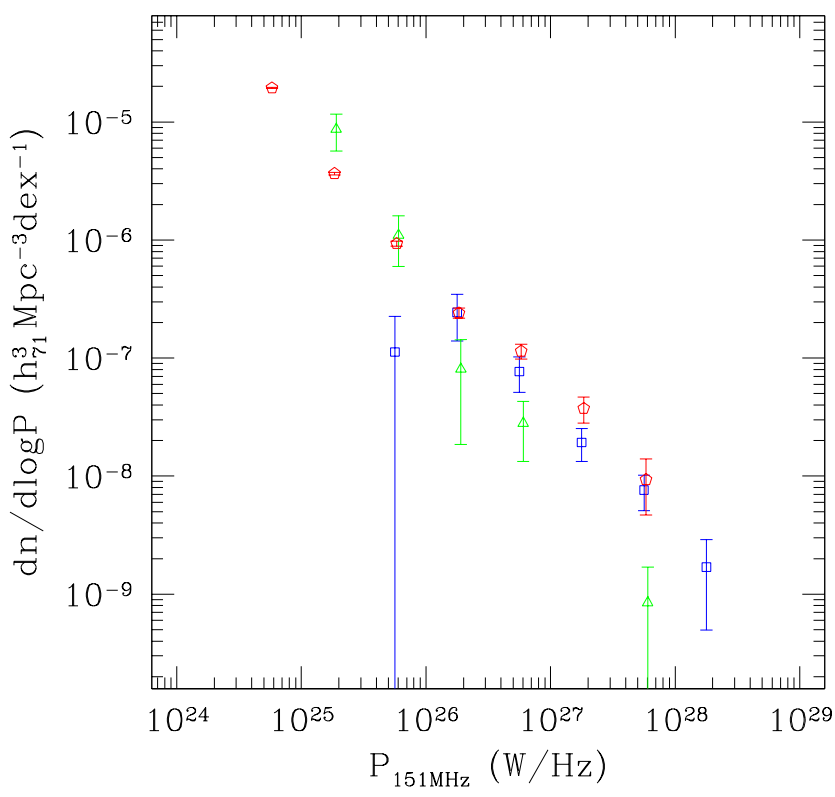

FIG. 8. - The $151 \mathrm{MHz}$ RLF at $z \leq 0.3$ constructed from the 3CRR survey, using data from Laing et al. (1983). The blue squares are for FR IIs, and the green triangles are for FR Is. The red pentagons are the model prediction (sum of the two populations).

Unlike W08, we do not consider radio-quiet AGNs and star-forming galaxies in the radio source model. As our primary goal is to simulate the microwave sky at relatively bright flux limits (e.g., $\gtrsim 0.1 \mathrm{mJy}$ ), ignoring these populations has negligible effects. Furthermore, the infrared source modeling presented in $\$ 2.5$ is a self-contained model for the starforming galaxies, and therefore the combined infrared and radio source models should adequately account for the majority of sources of interest to the current generation of microwave experiments.

Our model is a way to populate dark matter halos with radio sources in a Monte Carlo fashion, according to simple prescriptions on (1) the spectral energy distribution of the sources, (2) the number of radio sources as a function of halo mass and redshift, and (3) the (radio) luminosity distribution (LD) of the sources. Below we outline the most important aspects of the model. 


\subsubsection{Compact versus extended emission of radio sources}

The extended lobes of radio sources are characterized by a steep falling spectrum. On the other hand, the emission from the core region of a radio source is usually flatter and can exhibit a more complex spectral shape (see e.g., Lin et al. $2009 \mathrm{~b})$. In addition, when the jet axis is close to the observer's line-of-sight, the fluxes from the core (and/or the base of the jets) will be Doppler-boosted.

Following W08, we assume the observed core-to-lobe flux ratio, $R_{\mathrm{obs}}$, is related to the intrinsic value, $R_{\mathrm{int}}$, via $R_{\mathrm{obs}}=$ $R_{\text {int }} B(\theta)$, where $\theta$ is the angle between the jet axis and the line-of-sight, $B(\theta)=\left[(1-\beta \cos \theta)^{-2}+(1+\beta \cos \theta)^{-2}\right] / 2, \beta=$ $\sqrt{1-\gamma^{-2}}$, and $\gamma$ is the Lorentz factor of the jet.

Given the intrinsic luminosity $L_{\mathrm{int}}=L_{\mathrm{c} \text {,int }}+L_{\mathrm{l} \text {,int }}$ of a source, where the subscripts $c$ and 1 refer to the core and lobes, the beamed luminosity is then

$$
\begin{aligned}
L_{\text {beam }} & =L_{\mathrm{c}, \text { beam }}+L_{1, \text { int }} \\
& =\left(1+R_{\text {obs }}\right) L_{1, \text { int }} \\
& =L_{\text {int }}\left(1+R_{\text {obs }}\right) /\left(1+R_{\text {int }}\right)
\end{aligned}
$$

We use empirically derived values of $R_{\text {int }}$ and $\gamma$ from W08, and we assume a uniform distribution of $\cos \theta$ in order to separate $L_{\mathrm{c} \text {,beam }}$ and $L_{1, \text { int }}$ given $L_{\text {beam }}$.

Since the compact core emission is usually observed to exhibit a complex spectral shape (Lin et al. 2009b), we model it with a third-order polynomial, $\log f_{\nu}=\sum_{i}^{3} a_{i}(\log \nu)^{i}$, where $\nu$ is in GHz. We assume the extended sources obey $f_{\nu} \propto \nu^{-0.8}$, which is also consistent with observations (Lin et al. 2009b).

\subsubsection{Halo occupation number and the $151 \mathrm{MHz} R L F$}

Our first task is to reproduce the local $151 \mathrm{MHz}$ radio luminosity function (RLF), which is effectively a convolution of the halo mass function, the halo occupation number, and the radio luminosity distribution. The main reason for targeting the RLF at $151 \mathrm{MHz}$ is because at such low frequencies, one can safely assume that the fluxes are mostly dominated by the steep spectrum, extended sources, and the resulting RLF is not very sensitive to biases due to orientation effects. In addition, this RLF is not greatly affected by synchrotron/inverse Compton losses (Blundell et al. 1999). For both FR I and FR II populations, we assume their halo occupation number (HON) is given by $N(M)=N_{0}\left(M / M_{0}\right)^{\alpha}$. As for the LD, we assume it is independent of halo mass, and adopt two-segment power-laws that roughly mimic the observed RLF:

$$
p(L)= \begin{cases}\left(L / L_{b}\right)^{m} & \text { if } L>L_{b} \\ \left(L / L_{b}\right)^{n} & \text { otherwise }\end{cases}
$$

We explore the parameter space spanned by the HON and LD for both radio source populations, until the combined RLF fits the observed one at $z \sim 0.1$, which is based on the 3CRR survey (Laing et al. 1983). A comparison of the model prediction and the observed $151 \mathrm{MHz}$ RLF is shown in Fig. 8 .

\subsubsection{Redshift evolution and source number counts}

Once our model successfully reproduces the low redshift $151 \mathrm{MHz}$ RLF, the radio sources are divided into two populations, roughly corresponding to the type I and II classification of Fanaroff \& Riley (1974). These populations are given different number density evolutions with redshift, so that both

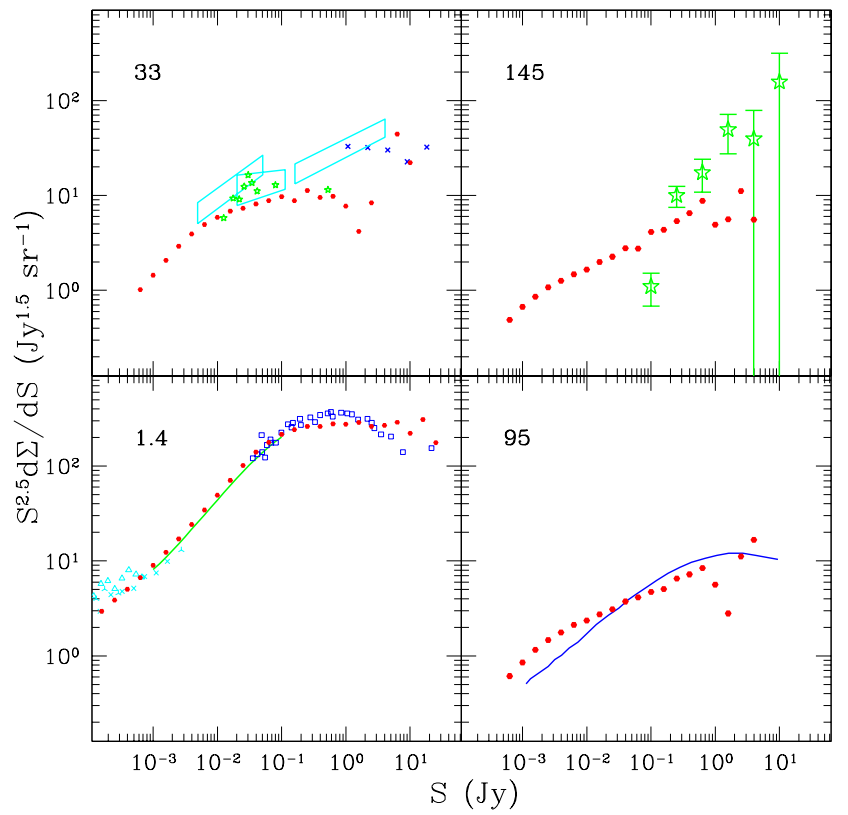

FIG. 9.- Source counts at four frequencies $(1.4,33,95$, and $145 \mathrm{GHz})$. The model prediction (one realization of $a_{i}$ 's - see Table 2] is shown as red solid points. All other symbols are observed source counts. The only exception is the curve in the $95 \mathrm{GHz}$ panel, which is itself a model from de Zotti et al. (2005), as shown in Sadler et al. (2008). The data used in the $1.4 \mathrm{GHz}$ panel are from Katgert et al. (1988); Windhorst et al. (1993); Bondi et al. (2003); Huynh et al. (2005). The data for $33 \mathrm{GHz}$ is from Wright et al. (2009); Cleary et al. (2005). The data for $145 \mathrm{GHz}$ is from ACBAR (Reichardt et al. 2009).

the high- $z$ RLFs and source counts at $151 \mathrm{MHz}$ can be fitted. For FR I sources, we consider an evolution of the form $(1+z)^{\delta}$ up to $z_{p}$ (and constant at $z>z_{p}$ ); for FR IIs, we find that a much stronger redshift evolution is needed and, for convenience, adopt an asymmetric Gaussian centered at $z_{p}$, with dispersions $\sigma_{l}$ and $\sigma_{h}$ at $z \leq z_{p}$ and $z>z_{p}$, respectively. (Note that there are many more FRIs and FRIIs at high redshift; for example, the comoving density of model FRII sources is $\sim 200$ times higher at $z_{p}=1.3$ compared to at $z \sim 0$.) In our model, $z_{p}$ is a free parameter, which is adjusted to match observations.

Having matched the high- $z$ 151 MHz RLF, we assume each source at $151 \mathrm{MHz}$ is described by $L_{\text {beam }}=L_{\mathrm{c} \text {,beam }}+L_{1, \text { int }}$, where the lobe component dominates. Given $R_{\text {int }}$ and $\gamma$ from W08, and $\cos \theta$ picked from a uniform distribution, we determine $L_{\text {c,beam. }}$. We next adjust the shape of the core SEDs (by varying the coefficients $a_{i}$ ) so that the source counts at $\nu>151 \mathrm{MHz}$ are reproduced. These source counts at higher frequencies are dominated by the radio cores. In Fig. 9, we show a comparison of our model prediction (red solid points) with observed source counts at $1.4,33,95$, and $145 \mathrm{GHz}$ (see caption for observation references). In general the model provides a good fit to the data ${ }^{12}$. We note that for the $95 \mathrm{GHz}$ panel, we are comparing our model to the predictions of the de Zotti et al. (2005) model.

There are 24 free parameters in the model, which fit $24 \mathrm{ob}-$ servational constraints (12 RLFs at various frequencies and redshifts, and 12 source count observations at 12 frequencies). (Note that $a_{0}, a_{1}, a_{2}, a_{3}$, and $\alpha$ are set to be the same for both

12 We have also compared our source count predictions with observations at $0.15,0.6,2.7,4.9,8.4,15,20,41$, and $61 \mathrm{GHz}$, and found good agreement. 
TABLE 2

MOdel PARAMETERS FOR RADIO SOURCES

\begin{tabular}{|c|c|c|c|c|c|c|c|c|c|c|c|c|c|c|c|c|}
\hline Type & $R_{\text {int }}$ & $\gamma$ & $a_{0}$ & $a_{1}^{\dagger}$ & $a_{2}^{\dagger}$ & $a_{3}^{\dagger}$ & $N_{0}$ & $\begin{array}{c}M_{0} \\
\left(h_{71}^{-1} M_{\odot}\right)\end{array}$ & $\alpha$ & $\begin{array}{c}L_{b} \\
(\mathrm{~W} / \mathrm{Hz})\end{array}$ & $m$ & $n$ & $\delta$ & $z p$ & $\sigma_{l}$ & $\sigma_{h}$ \\
\hline FR I & $10^{-2.6}$ & 6 & 0 & $(-0.12,0.07)$ & $(-0.34,0.99)$ & $(-0.75,-0.23)$ & 1 & $4 \times 10^{13}$ & 0.1 & $10^{24}$ & -1.55 & 0 & 3 & 0.8 & $\ldots$ & $\ldots$ \\
\hline FR II & $10^{-2.8}$ & 8 & 0 & $(-0.12,0.07)$ & $(-0.34,0.99)$ & $(-0.75,-0.23)$ & 0.015 & $3 \times 10^{15}$ & 0.1 & $10^{27.5}$ & -1.6 & -0.65 & $\cdots$ & 1.3 & 0.4 & 0.73 \\
\hline
\end{tabular}

$\dagger$ We draw random uniform variates in this range when assigning the spectral shape for cores.

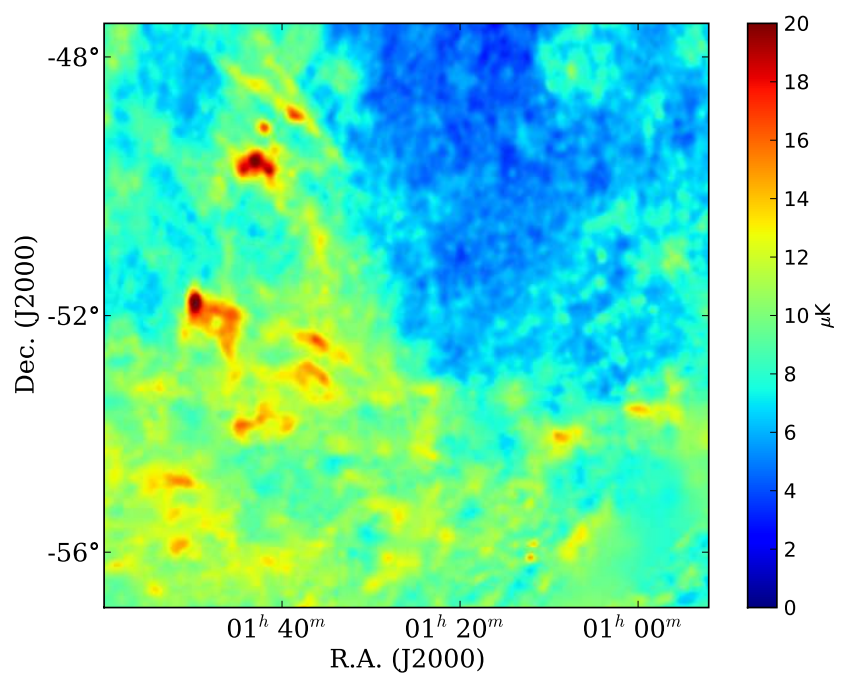

FIG. 10. - The simulated Galactic dust emission in a $\sim 10$ degree $\times 10$ degree patch.

FRIs and FRIIs.) Several of the parameters are degenerate; in Table 2 we present a parameter combination that can reproduce the results presented here.

For a more complete description of the model and further comparison with available observational constraints see Lin et al. (2009a).

\subsection{Galactic Emission}

For the contribution of Galactic thermal dust emission, we use the "model 8" prediction from Finkbeiner et al. (1999), an extrapolation to microwave frequencies of dust maps from Schlegel et al. (1998). We use the software included with these maps to interpolate onto an oversampled HEALPix grid with $N_{\text {side }}=8192$. This model is a two-component fit to IRAS, DIRBE, and FIRAS data, and is shown by Bennett et al. (2003) to be a reasonable template for dust emission in the WMAP maps.

The Galactic synchrotron and free-free emission is comparable to the Galactic dust emission at $\sim 70 \mathrm{GHz}$ and becomes dominate/subdominant at lower/higher frequencies (see e.g. Smoot 1998). Thus these signals are not expected to be a significant contaminant for ACT or SPT (which surveys target regions away from the Galaxy), although they will be important to consider for the Planck low-frequency channels. We leave the inclusion of these signals to later work, and note work by, for example, Leach et al. (2008), which discusses the inclusion of these signals in microwave simulations for Planck.

\subsection{General Map Properties}

Below we list some general properties of the maps themselves. The full-sky HEALPix maps for this simulation are in the Celestial coordinate system with RING ordering (Górski et al. 2005). The conversion between the HEALPix $\theta$ and $\phi$ coordinates and right ascension and declination is: $\phi=\mathrm{ra}, 90-\theta=\mathrm{dec}$. We store all maps in units of flux per solid angle (as Jy/sr). To convert to temperature (as $\mu \mathrm{K}$ ) we use the conversion:

$$
\frac{d T}{d I}=\frac{c^{2}}{2 k_{B} \nu^{2}} \frac{\left(e^{x}-1\right)^{2}}{x^{2} e^{x}}
$$

where $x=h \nu / k_{B} T_{C M B}$. To convert from SI to more convenient units requires $\mu \mathrm{K} \mathrm{sr} / \mathrm{Jy}=10^{20} \mathrm{~K} \mathrm{sr} \mathrm{m}{ }^{2} \mathrm{~Hz} / \mathrm{W}$. When calculating the power spectrum with Polspice ${ }^{13}$ or anafast ${ }^{14}$, a correction for the pixel window function is required.

The properties of the halos that are specified in the halo catalog are redshift, ra, dec, position of the halo potential minimum, peculiar velocity, friends-of-friends mass, virial mass, virial gas mass, virial radius, integrated Compton $y$ parameter, integrated kinetic SZ, integrated full SZ at the six frequencies (the latter three integrated within the virial radius, $R_{200}$, and $R_{500}$ ), the stellar mass, and the central gas density, temperature, pressure, and potential. In the radio and infrared galaxy catalogs, the properties given for each source are redshift, ra, $\mathrm{dec}$, and the flux in mJy at the six frequencies.

The large-scale structure octant is mirrored into full-sky maps as follows:

$$
\begin{aligned}
\theta_{N} & =\theta_{0} \\
\phi_{N} & =\phi_{0}+n \frac{\pi}{2} \\
\theta_{S} & =\pi-\theta_{0} \\
\phi_{S} & =\frac{\pi}{2}-\phi_{0}+n \frac{\pi}{2}
\end{aligned}
$$

where $n=0,1,2$, and 3, and the subscripts $N$ and $S$ refer to the northern and southern hemispheres of the map. The subscript 0 refers to the coordinates in the original octant. For the power spectrum analysis, this method for repetition in the Southern hemisphere is preferable to reflecting the North. Although simple mirroring eliminates real space discontinuities across the equator, it makes the simulated sky an even function with respect to the polar axis. In the spherical harmonic transform, the associated Legendre functions are even or odd depending on $l$. Integrating mirrored hemispheres over them yields zero for odd- $l$ harmonic coefficients. Because rotating the sky transforms harmonics with common $l$ into each other,

\footnotetext{
13 http://www.planck.fr/article141.html

$14 \mathrm{http} / / /$ healpix.jpl.nasa.gov/html/facilitiesnode6.htm
} 
odd- $l$ harmonics will be zero regardless of the orientation of the mirroring.

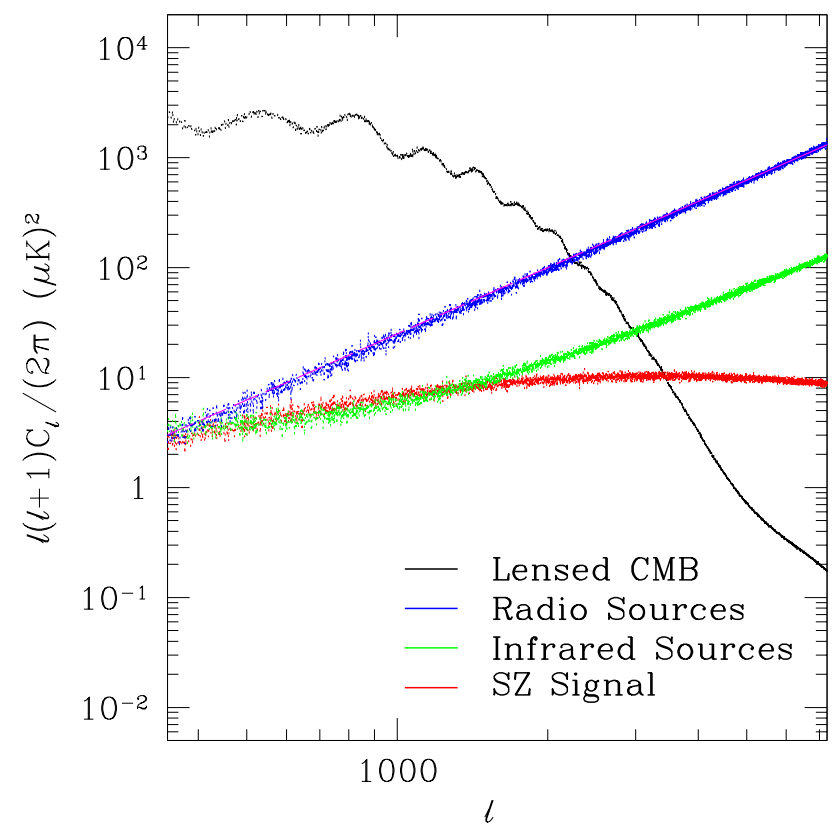

FIG. 11.- Power spectra of the lensed microwave background (black), radio galaxies (blue), infrared galaxies (green), and full Sunyaev-Zel'dovich signal (red) in this simulation at $148 \mathrm{GHz}$. The magenta line through the blue radio source power spectrum gives the radio source power from the Toffolatti et al. (1998) model scaled by 0.64, which was found by Wright et al. (2009) to be a good fit to the WMAP 5-year source counts.

\subsection{Power Spectra of Simulated Components}

In Fig. 11, we show the power spectra of the lensed microwave background, infrared galaxies, radio galaxies, and the full SZ signal from the simulated maps at $148 \mathrm{GHz}$. The SZ power peaks at around $l \sim 3000$ and the clustering of the infrared galaxies is apparent for $l<1000$, consistent with measurements by Spitzer (Lagache et al. 2007) and BLAST (Viero et al. 2009). No infrared or radio sources have been removed in creating the power spectra, which were made with Polspice on the full-sky maps. The power from the infrared and radio sources, without masking out bright sources, is comparable to the lensed microwave background between $l \sim 2000$ and $l \sim 3000$. In Fig. 11, the magenta line through the blue radio source power spectrum gives the radio source power suggested by the Toffolatti et al. (1998) model scaled by 0.64 , which was found by Wright et al. (2009) to be a good fit to the WMAP 5-year source counts. The two power spectra are in excellent agreement.

\subsection{Y-M Relation and Scatter}

We investigate the SZ flux versus cluster mass scaling relation for this simulation below. An accurate calibration of this relation off observations is important for assessing the selection function of a potential SZ selected cluster sample via simulations. The gas physics input into the simulations will determine the surface brightness of a given cluster based on its mass and redshift, which in turn determines how likely it is to be detected by a given observation strategy.
To characterize the SZ flux - cluster mass scaling relation in this simulation we compare the integrated Compton $y$ parameter to cluster mass for the halos in the one unique octant. The integrated Compton $y$ parameter is given by $Y=\left(\int y d \Omega\right) d_{A}^{2}(z)$, where $y$ is defined in Eq. 2, $d \Omega$ is the solid angle of the cluster, and $d_{A}^{2}(z)$ is the cluster's angular diameter distance. For the self-similar model, we expect a virialized halo of mass $M_{\text {vir }}$ to have a virial temperature of

$$
T_{\mathrm{vir}} \propto\left[M_{\mathrm{vir}} E(z)\right]^{2 / 3},
$$

where for a flat $\Lambda$ CDM cosmology

$$
E(z)=\left[\Omega_{m}(1+z)^{3}+\Omega_{\Lambda}\right]^{1 / 2} .
$$

If galaxy clusters were isothermal, then the Compton $y$ parameter would satisfy the relation

$$
Y \propto f_{\text {gas }} M_{\text {halo }} T
$$

where $f_{\text {gas }}$ is the cluster gas mass fraction. Combining the above equations, we would expect

$$
Y \propto f_{\mathrm{gas}} M_{\mathrm{vir}}^{5 / 3} E(z)^{2 / 3},
$$

for the self-similar relation. Deviations from the self-similar relation are not unexpected as clusters are not isothermal, not always in virial equilibrium, and $f_{\text {gas }}$ need not be constant with variations in cluster mass and redshift.

The gas physics in this simulation has been calibrated off of X-ray observations of cluster gas fractions as a function of temperature as discussed in $\$ 2.4$ and Bode et al. (2009). For each halo with $M_{\mathrm{FoF}}>2 \times 10^{13} h^{-1} M_{\odot}$ and $z<3$ (whose gas model is described in $\$ 2.4 .1$ we calculate $Y_{200}$ and $Y_{500}$, which are the projected Compton $y$ parameter in a disk of radius $R_{200}$ and $R_{500}$ respectively. Here $R_{200}$ and $R_{500}$ are the radii within which the cluster mean mass density is 200 and 500 times the critical density at the cluster redshift. We then rank the clusters by mass and bin them in mass bins of $\Delta \log \left(\mathrm{M}_{200}\right)=$ 0.05 and $\Delta \log \left(\mathrm{M}_{500}\right)=0.1$ for $M_{200}$ and $M_{500}$ respectively. The mean of $M_{\Delta}$ as well as the mean and standard deviation on the mean of $Y_{\Delta} \times E(z)^{-2 / 3}$ are then calculated for each bin. These values, for $M_{200}$ and $Y_{200}$, are plotted in Fig. 12, along with all the individual halos with $M_{200}>2 \times 10^{14} M_{\odot}$ in the octant.

The binned values are then fit to the power-law relation

$$
\frac{Y_{\Delta}}{E(z)^{2 / 3}}=10^{\beta}\left(\frac{M_{\Delta}}{10^{14} M_{\odot}}\right)^{\alpha} .
$$

For $M_{200}>2 \times 10^{14} M_{\odot}$, we find for the $Y_{200}$ versus $M_{200}$ relation $\alpha=1.682 \pm 0.004$ and $\beta=-5.648 \pm 0.002$, with a reduced $\chi^{2}$ of 1.2 for $\sim 13,000$ clusters divided into 19 bins. This slope is slightly steeper than that expected for the self-similar case. It is also consistent with the $Y-M$ relations derived from hydrodynamical cluster simulations (e.g. White et al. 2002; da Silva et al. 2004; Motl et al. 2005; Nagai 2006; Shaw et al. 2008). The $Y-M$ relation calculated within a disk of radius $R_{2500}$ is also consistent with the observed $Y_{2500}-M_{\text {gas }}\left(<R_{2500}\right)$ relation of Bonamente et al. (2008), as shown in Bode et al. (2009). 
TABLE 3

Best- Fit Parameters for the $Y_{\Delta}-M_{\Delta}$ Relation, With $M_{\Delta}>2 \times 10^{14} M_{\odot}$, FitTed USING THE POWER LAW Given IN EQUATION 33

\begin{tabular}{ccccccccc}
\hline \hline Relation & $\alpha$ & $\sigma_{\alpha}$ & $\beta$ & $\sigma_{\beta}$ & Reduced $\chi^{2}$ & Clusters & Bins & $\sigma_{Y M}$ \\
\hline$Y_{200}-M_{200}$ & 1.682 & 0.004 & -5.648 & 0.002 & 1.2 & 12970 & 19 & 0.14 \\
$Y_{500}-M_{500}$ & 1.668 & 0.009 & -5.509 & 0.004 & 1.9 & 4900 & 8 & 0.17 \\
$Y_{500}-M_{200}$ & 1.693 & 0.004 & -5.765 & 0.002 & 1.8 & 12970 & 19 & 0.13 \\
\hline
\end{tabular}

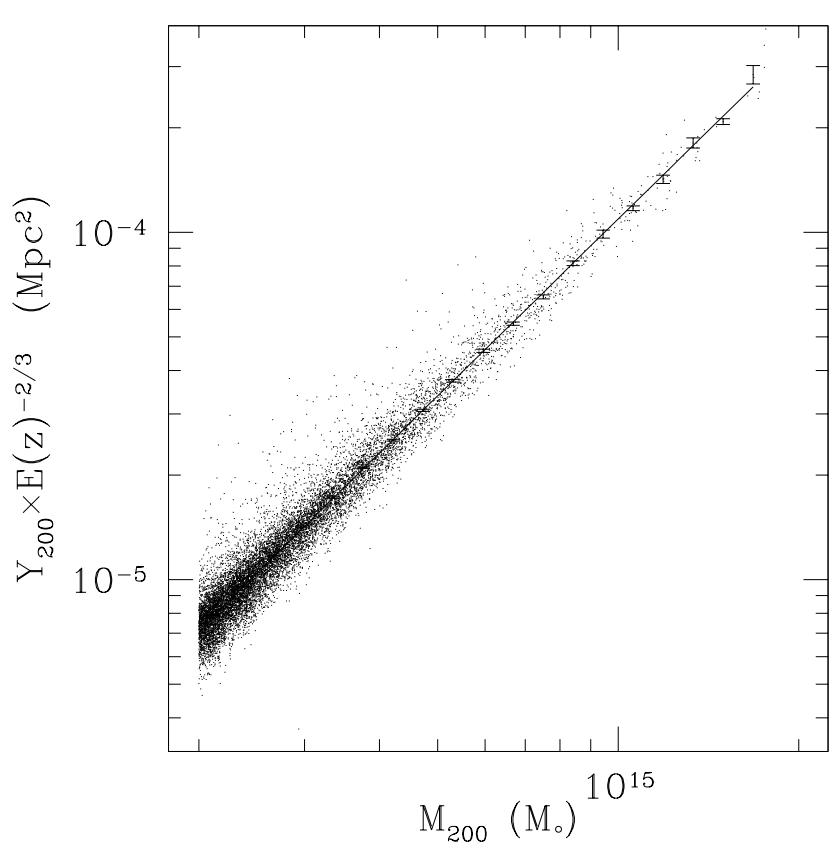

FIG. 12. $-Y_{200}$ versus $M_{200}$ relation for all the halos from one octant of this simulation with $M_{200}>2 \times 10^{14} M_{\odot}$. The points with error bars represent bins of width $\Delta \log \left(\mathrm{M}_{200}\right)=0.05$ and errors on the bin mean. The best-fit parameters for this scaling relation and the scatter are given in Table 3

We also find the scatter in this $Y-M$ relation about the bestfit relation. Specifically we calculate

$$
\sigma_{Y M}=\left[\frac{\sum_{i=1}^{N}\left(\ln \tilde{\mathrm{Y}}_{\Delta}-\ln \mathrm{Y}_{\Delta}\right)_{\mathrm{i}}^{2}}{N-2}\right]^{1 / 2}
$$

as discussed in e.g. Shaw et al. (2008), where $\tilde{Y}_{\Delta}$ is the projected SZ flux measured within a disk of radius $R_{\Delta}, Y_{\Delta}$ is the fitted SZ flux of for a cluster of mass $M_{\Delta}$ using the best-fit parameters for Eq. 33, and $\mathrm{N}$ is the total number of clusters. We find a scatter for the $Y_{200}-M_{200}$ relation of about $14 \%$, and a slightly larger scatter of about $17 \%$ for the $Y_{500}-M_{500}$ relation. We find a lower scatter of roughly $13 \%$ for the $Y_{500}-M_{200}$ relation, which has been suggested by Shaw et al. (2008) to have less scatter than the $Y_{200}-M_{200}$ relation. We list the bestfit parameters for Eq. 33 and the scatter for the $Y_{200}-M_{200}$, $Y_{500}-M_{500}$, and $Y_{500}-M_{200}$ relations in Table 3 .

Concerning the scatter given above, we assume hydrostatic equilibrium for the gas; however, we do not assume the same entropy level for all the halos. The kinetic energy of the gas is derived from that of the dark matter, so for a non-virialized, merging halo with a high kinetic energy relative to the cluster potential, the gas will also be given a high energy and thus temperature. Thus merging of halos is taken into account at some level, although a full treatment of the gas physics would give more scatter. Also, the repetition of halos as the periodic box is tiled to fill the octant, even though these halos are often at different redshifts and evolutionary states, may serve to slightly decrease the scatter.

Note that the $Y$ values discussed above are intrinsic to the cluster and do not include any contamination from foreground or background SZ signals along the line of sight. Inclusion of this projection contamination will increase the $Y-M$ scatter somewhat, but for clusters with masses greater than roughly $2 \times 10^{14} M_{\odot}$, an optimistic mass limit of current SZ surveys, this projection contamination should be below $\sim 20 \%$ over a wide range of possible $\sigma_{8}$ values $(0.7-1.0)$ and redshifts $(z<$ 2) (Holder et al. 2007; Hallman et al. 2007).

\subsection{Point Source Contamination of SZ Clusters}

An important question for both determinations of an SZ cluster sample's selection function and measurements of the SZ power spectrum is the correlation and contamination of the SZ cluster signal by infrared and radio galaxies. Observations suggest we should expect some preference for radio and infrared galaxies to reside in galaxy clusters (e.g. Coble et al. 2007; Lin \& Mohr 2007; Daddi et al.2009), and these sources could potentially fill in the SZ decrement at frequencies below the null. This point source contamination of the SZ flux could both affect cluster detection and bias a measured SZ flux mass scaling relation. If these effects are significant and unaccounted for, then inaccurate cosmological conclusions would result from measured SZ cluster samples. Regarding measurements of the SZ power spectrum and derived values of $\sigma_{8}$, typically a significant number of high flux point sources are masked out of microwave maps before determining the power spectrum. If these sources reside preferentially in massive clusters, a significant portion of the SZ signal could be masked out and a biased determination of $\sigma_{8}$ would arise.

Here we investigate, via these simulations, the fraction of SZ clusters that may be substantially contaminated by radio or infrared galaxy populations. We first add the flux of all galaxies of a given population residing in a given halo. Then, using each galaxy cluster's integrated SZ flux within $R_{200}$ (in units of mJy), we calculate the fraction of halos whose combined flux from resident galaxies equals $20 \%$ or more of the halo's SZ flux decrement at 30, 90, and $148 \mathrm{GHz}$. Such halos will be harder to detect by SZ surveys, and their signal is more likely to be masked out in attempts to measure the SZ power spectrum. We choose a $20 \%$ contamination fraction as that is roughly where the uncertainty in the expected SZ flux for a cluster of a given mass becomes dominated by point source contamination as opposed to the intrinsic scatter in the $Y-M$ relation or projection effects.

For the contamination due to radio sources, we find that for halos with $M_{200}>1 \times 10^{14} M_{\odot}$, roughly $3 \%$ or less of clusters with $z<1.6$ have their SZ flux decrement at $148 \mathrm{GHz}$ filled in by more than $20 \%$ (see Fig. 13). At $90 \mathrm{GHz}$, this fraction increases slightly, and less than $4 \%$ of clusters with $z<1.6$ 


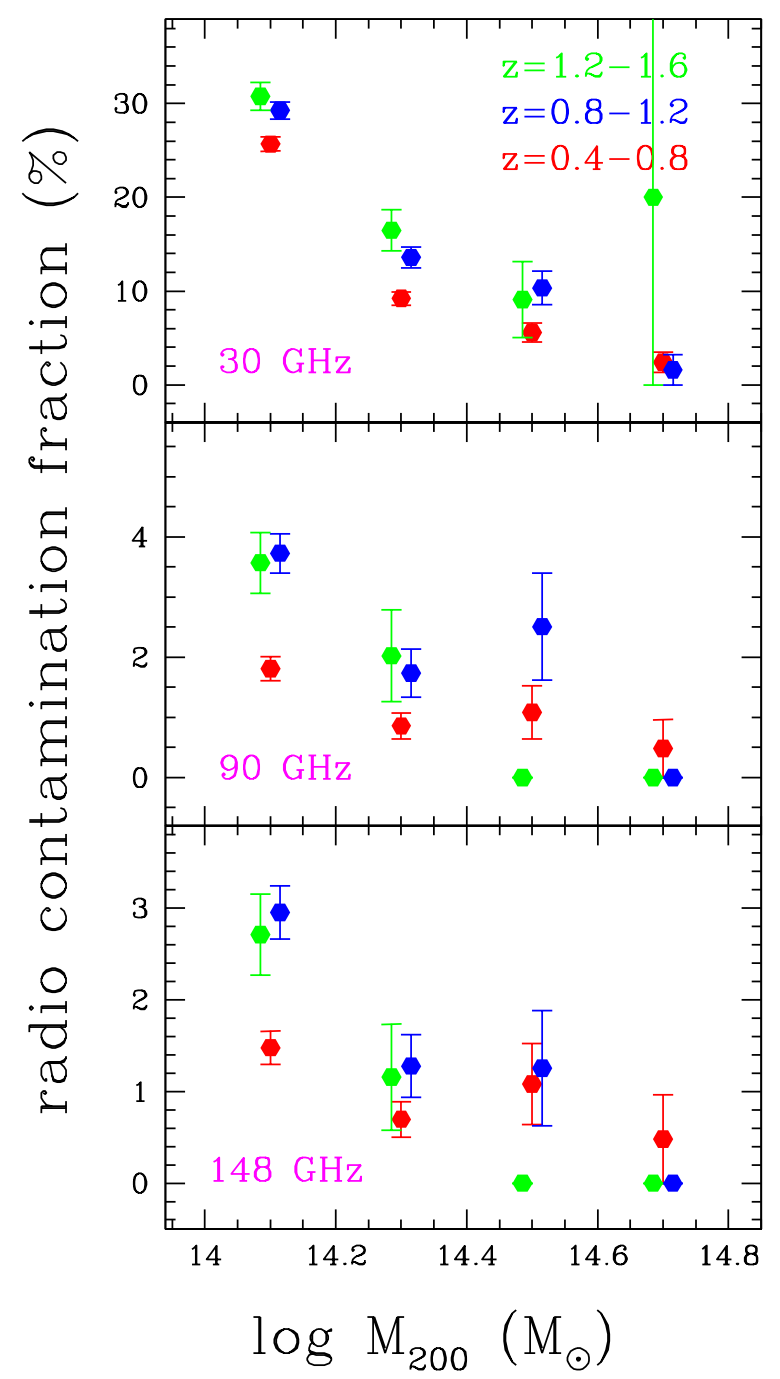

FIG. 13. - Fraction of halos in this simulation that host radio galaxies whose combined flux equals $20 \%$ or more of the halo's SZ flux decrement at $30 \mathrm{GHz}$ (top), $90 \mathrm{GHz}$ (middle), and $148 \mathrm{GHz}$ (bottom). Bin widths are $\Delta \log \left(\mathrm{M}_{200}\right)=0.2$, and the error bars assume a Poisson distribution. Red, blue, and green dots indicate the redshift ranges of $0.4-0.8,0.8-1.2$, and $1.2-1.6$ respectively.

have a contamination of $20 \%$ or more. At $30 \mathrm{GHz}$, one may expect 20 to $30 \%$ of clusters with $M_{200} \sim 1 \times 10^{14} M_{\odot}$ to be so contaminated, with a decrease in this fraction as the halo mass is increased. These contamination fractions exhibit a weak redshift dependence, with slightly larger fractions at higher redshifts.

Infrared galaxies seem to be a significant source of contamination for SZ clusters at 90 and $148 \mathrm{GHz}$ (see Figs. 14 and 15). However, these simulations suggest that, at $90 \mathrm{GHz}$, the fraction of halos with $M_{200}>2.5 \times 10^{14} M_{\odot}$ and $z<1.2$, that are contaminated at a level of $20 \%$ or more, is less than $20 \%$. In all mass bins, the general trend is for the contamination fraction to increase at higher redshifts where more infrared galaxies reside. There is no appreciable contamination from infrared galaxies at $30 \mathrm{GHz}$. At $148 \mathrm{GHz}$, less than $20 \%$ of halos with $M_{200}>2.5 \times 10^{14} M_{\odot}$ and $z<0.8$ have their SZ decrements filled in at a level of $50 \%$ or more (see Fig. (15). It should be noted that the above estimates only include galaxies that reside in halos and does not include contamination from galaxies along a halo's line of sight. In this regard, these fractions should be viewed as lower limits. The one exception to this is for the very high flux infrared galaxies that most likely represent a lensed population of galaxies. These galaxies are placed in massive halos and are the main source of contamination in the most massive bins. These sources should more properly be placed behind massive halos, however, the contamination effect in projection will still be similar.

Such contamination fractions as discussed above should to be factored into sample completeness estimates and the scatter in the $Y-M$ relation in order to use SZ detected clusters for precision cosmology. The models presented here should give a reasonable approximation to the expected levels of contamination from the different galaxy populations. However, these estimates should be cross-checked and made more precise with multi-wavelength observations of SZ clusters.

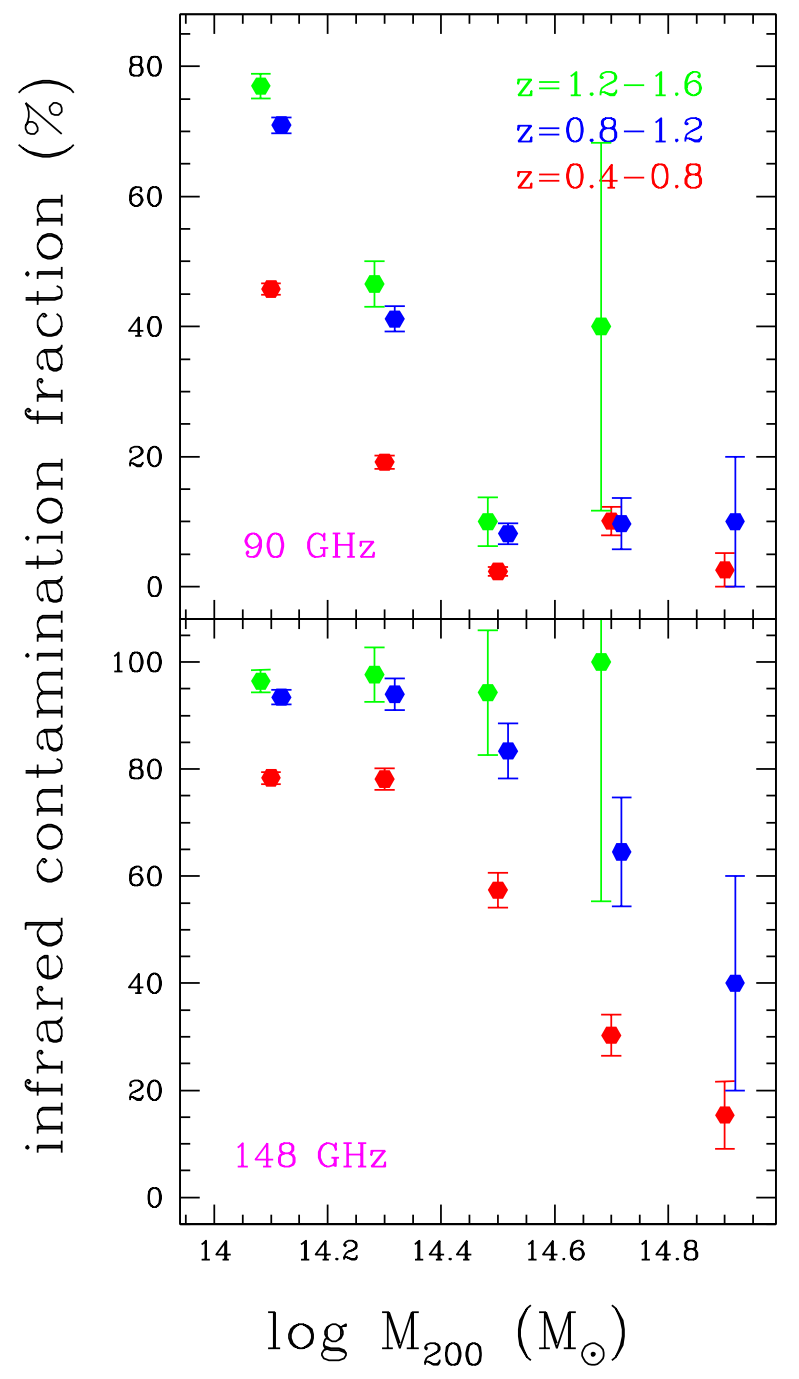

FIG. 14.- Fraction of halos in this simulation that host infrared galaxies whose combined flux equals $20 \%$ or more of the halo's SZ flux decrement at $90 \mathrm{GHz}$ (top) and $148 \mathrm{GHz}$ (bottom). Bin widths and redshift ranges are the same as in Fig.13 An absence of an indicated fraction indicates no halos in that mass and redshift interval. 


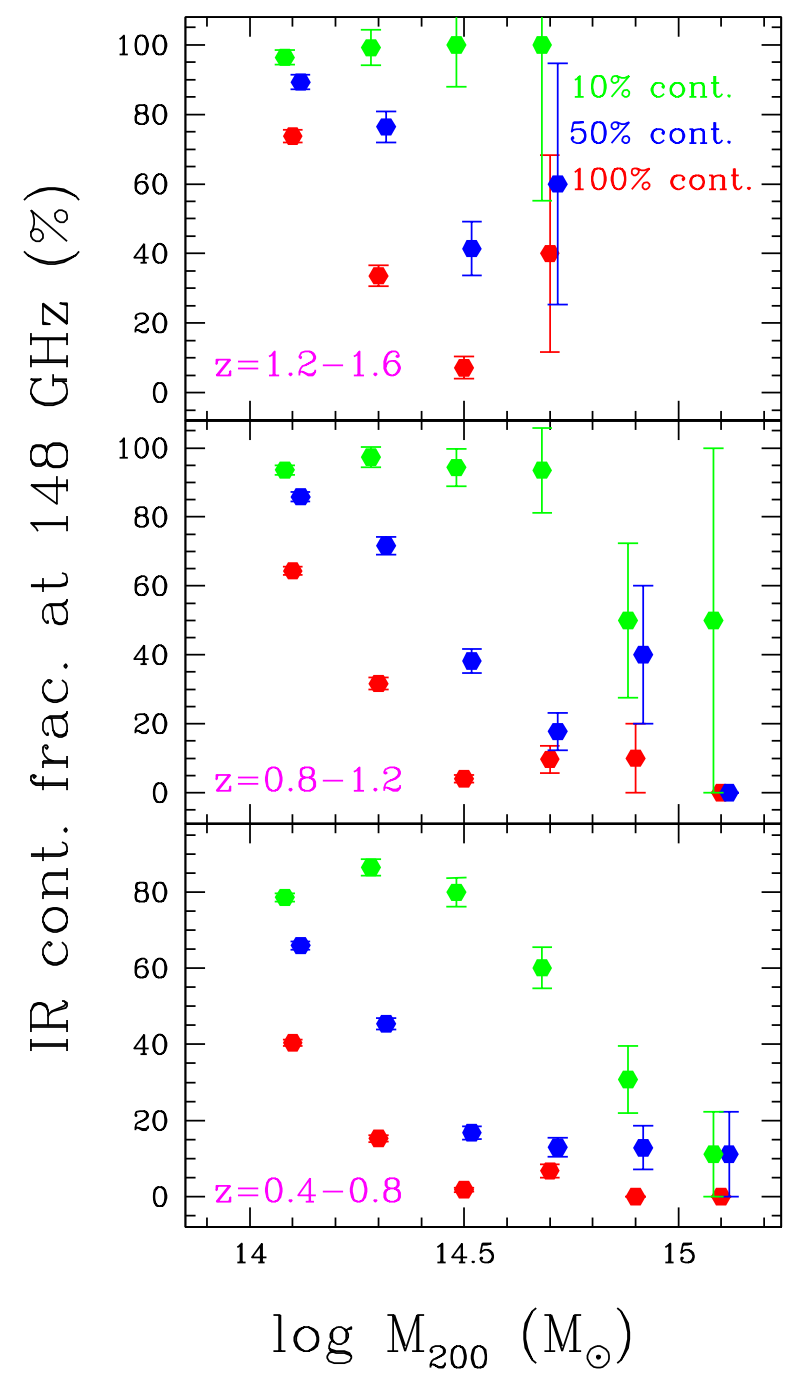

FIG. 15.- Fraction of halos in this simulation that host infrared galaxies whose combined flux is greater than $10 \%$ (green), $50 \%$ (blue), or $100 \%$ (red) of the halo's SZ flux decrement at $148 \mathrm{GHz}$. Bin widths are the same as in Fig. 13 This is shown for the redshift ranges of 1.2-1.6 (top), 0.8-1.2 (middle), and $0.4-0.8$ (bottom).

\section{CONCLUSION}

The simulations discussed here have been created for the use of the ACT team to test their data analysis pipeline. To this end, we have modeled the primary astrophysical signals as realistically as possible by matching closely to recent observations. We have also expanded the frequency coverage beyond the ACT bands and created maps and catalogs in an easily accessible format. As such, other current and upcoming microwave background experiments may find these simulations useful for a variety of purposes.

From the gas prescription in these simulations, which has been matched to recent X-ray observations, we find a scaling relation between Compton $y$ parameter and mass that is only slightly steeper than self-similar and has a scatter of $14 \%$ to $17 \%$ around the best-fit relation for $Y_{200}-M_{200}$ and $Y_{500}-M_{500}$ respectively. This low intrinsic scatter suggests that measurement of the cluster SZ flux may be a relatively clean cluster mass proxy. The correlation of radio galaxies with SZ clusters suggests that at $148 \mathrm{GHz}(90 \mathrm{GHz})$, for clusters with $M_{200}>10^{14} M_{\odot}$, less than $3 \%(4 \%)$ of these clusters will have their SZ decrement filled in by $20 \%$ or more. We find the contamination levels higher for infrared galaxies. However, at 90 $\mathrm{GHz}$, less than $20 \%$ of clusters with $M_{200}>2.5 \times 10^{14} M_{\odot}$ and $z<1.2$ have their SZ decrements filled in at a level of $20 \%$ or more. At $148 \mathrm{GHz}$, less than $20 \%$ of clusters with $M_{200}>2.5 \times 10^{14} M_{\odot}$ and $z<0.8$ have their SZ decrements filled in at a level of $50 \%$ or larger. We also find that a population of very high flux infrared galaxies, which may have been lensed, contribute most to the SZ contamination of very massive clusters at 90 and $148 \mathrm{GHz}$. Multi-wavelength observations of SZ clusters will help to make these contamination estimates more precise. This information is crucial for determining SZ cluster selection functions and the actual scatter in the observed $Y-M$ relation, which in turn are vital to efforts to extract cosmology from SZ cluster counts. The inclusion of the lensing of the microwave background in a manner internally consistent with the large and small-scale structure is necessary to verify the accurate recovery of cosmology from cross-correlation studies between the lensed microwave background and tracers of large-scale structure. Moreover, the simulations as a whole, matched to the most recent observations, will inform, through a variety of means, measurement of the angular power spectrum, higher point correlation functions, and the cosmology thus derived.

Cosmic microwave background experiments are entering an exciting time of finer resolution and higher sensitivity which will open up the Sunyaev-Zel'dovich Universe, microwave background lensing, an assortment of cross-correlation studies, and deeper probes of inflation through tighter parameter constraints. These simulations should provide a useful tool to aid analyses in these new and promising areas of discovery.

NS thanks Steve Allen, Olivier Dore, Joanna Dunkley, Joe Fowler, Gil Holder, Andrew Lawrence, Toby Marriage, Danica Marsden, Lyman Page, and David Spergel for useful discussions. SD thanks David Spergel, Chris Hirata, Paul Bode and Hy Trac for help and guidance during the development of the original lensing code. CHM is grateful to R.E. Smith for insightful comments on the power spectrum modeling. HT thanks Kavi Moodley and Ryan Warne for helpful discussions. NS is supported by the U.S. Department of Energy contract to SLAC no. DE-AC3-76SF00515. PB was partially supported by NSF grant 0707731. SD acknowledges support from NASA ATP Grant NNX08AH30G. YTL acknowledges support from the World Premier International Research Center Initiative, MEXT, Japan. HT is supported by the Institute for Theory and Computation Fellowship. Computer simulations and analysis were supported by the National Science Foundation through TeraGrid resources provided by the Pittsburgh Supercomputing Center and the National Center for Supercomputing Applications under grant AST070015; computations were also performed at the TIGRESS high performance computer center at Princeton University, which is jointly supported by the Princeton Institute for Computational Science and Engineering and the Princeton University Office of Information Technology. We acknowledge support from the PIRE program and NSF Grant OISE/0530095. Some of the results in this paper have been derived using the HEALPix package (Górski et al.2005). We also acknowledge the use of the Legacy Archive for Microwave Background Data Analysis (LAMBDA). Support for LAMBDA is provided by the 
NASA Office of Space Science.

\section{REFERENCES}

Acquaviva, V., Hajian, A., Spergel, D. N., \& Das, S. 2008, Phys. Rev. D, 78, 043514

Amblard, A., Vale, C., \& White, M. 2004, New Astronomy, 9, 687

Austermann, J. E., et al. 2009, ArXiv e-prints

Basak, S., Prunet, S., \& Benabed, K. 2008, ArXiv e-prints

Bennett, C. L., et al. 2003, ApJS, 148, 97

Blain, A. W. 1999, MNRAS, 309, 955

Blundell, K. M., Rawlings, S., \& Willott, C. J. 1999, AJ, 117, 677

Bode, P., \& Ostriker, J. P. 2003, ApJS, 145, 1

Bode, P., Ostriker, J. P., \& Vikhlinin, A. 2009, ArXiv e-prints

Bode, P., Ostriker, J. P., Weller, J., \& Shaw, L. 2007, ApJ, 663, 139

Bode, P., Ostriker, J. P., \& Xu, G. 2000, ApJS, 128, 561

Bonamente, M., Joy, M., LaRoque, S. J., Carlstrom, J. E., Nagai, D., \& Marrone, D. P. 2008, ApJ, 675, 106

Bondi, M., et al. 2003, A\&A, 403, 857

Carbone, C., Baccigalupi, C., Bartelmann, M., Matarrese, S., \& Springel, V. 2008, ArXiv e-prints

Carbone, C., Baccigalupi, C., Bartelmann, M., Matarrese, S., \& Springel, V. 2009, MNRAS, 576

Carbone, C., Springel, V., Baccigalupi, C., Bartelmann, M., \& Matarrese, S. 2007, ArXiv e-prints

Cen, R., \& Ostriker, J. P. 1999, ApJ, 514, 1

Cleary, K. A., et al. 2005, MNRAS, 360, 340

Coble, K., et al. 2007, AJ, 134, 897

Cohn, J. D., \& White, M. 2009, MNRAS, 393, 393

Coppin, K., et al. 2006, MNRAS, 372, 1621

da Silva, A. C., Kay, S. T., Liddle, A. R., \& Thomas, P. A. 2004, MNRAS, 348, 1401

Daddi, E., et al. 2009, ApJ, 694, 1517

Das, S., \& Bode, P. 2008, ApJ, 682, 1

Das, S., \& Spergel, D. N. 2009, Physical Review D (Particles, Fields, Gravitation, and Cosmology), 79, 043509

Davé, R., et al. 2001, ApJ, 552, 473

de Putter, R., Zahn, O., \& Linder, E. V. 2009, ArXiv e-prints

de Zotti, G., Ricci, R., Mesa, D., Silva, L., Mazzotta, P., Toffolatti, L., \& González-Nuevo, J. 2005, A\&A, 431, 893

Devlin, M. J., et al. 2009, Nature, 458, 737

Diaferio, A., et al. 2005, MNRAS, 356, 1477

Dunkley, J., et al. 2009, ApJS, 180, 306

Dunne, L., Eales, S., Edmunds, M., Ivison, R., Alexander, P., \& Clements, D. L. 2000, MNRAS, 315, 115

Dye, S., et al. 2009, ArXiv e-prints

Fanaroff, B. L., \& Riley, J. M. 1974, MNRAS, 167, 31P

Finkbeiner, D. P., Davis, M., \& Schlegel, D. J. 1999, ApJ, 524, 867

Fixsen, D. J., Dwek, E., Mather, J. C., Bennett, C. L., \& Shafer, R. A. 1998, ApJ, 508, 123

Gawiser, E., et al. 1998, ArXiv Astrophysics e-prints

Gawiser, E., \& Smoot, G. F. 1997, ApJ, 480, L1

Górski, K. M., Hivon, E., Banday, A. J., Wandelt, B. D., Hansen, F. K., Reinecke, M., \& Bartelmann, M. 2005, ApJ, 622, 759

Haiman, Z., \& Knox, L. 2000, ApJ, 530, 124

Hallman, E. J., O'Shea, B. W., Burns, J. O., Norman, M. L., Harkness, R., \& Wagner, R. 2007, ApJ, 671, 27

Hallman, E. J., O'Shea, B. W., Smith, B. D., Burns, J. O., \& Norman, M. L. 2009, ApJ, 698, 1795

Hernández-Monteagudo, C., Trac, H., Verde, L., \& Jimenez, R. 2006, ApJ, 652, L1

Hinshaw, G., et al. 2009, ApJS, 180, 225

Hirata, C. M., \& Seljak, U. 2003, Phys. Rev. D, 67, 043001

Holder, G. P., McCarthy, I. G., \& Babul, A. 2007, MNRAS, 382, 1697

Hopkins, A. M., \& Beacom, J. F. 2006, ApJ, 651, 142

Hu, W., \& Okamoto, T. 2002, ApJ, 574, 566

Huynh, M. T., Jackson, C. A., Norris, R. P., \& Prandoni, I. 2005, AJ, 130, 1373

Jackson, C. A., \& Wall, J. V. 1999, MNRAS, 304, 160

Jenkins, A., Frenk, C. S., White, S. D. M., Colberg, J. M., Cole, S., Evrard,

A. E., Couchman, H. M. P., \& Yoshida, N. 2001, MNRAS, 321, 372

Katgert, P., Oort, M. J. A., \& Windhorst, R. A. 1988, A\&A, 195, 21

Kennicutt, R. C., Jr. 1998, ARA\&A, 36, 189

Knox, L., Holder, G. P., \& Church, S. E. 2004, ApJ, 612, 96

Komatsu, E., et al. 2009, ApJS, 180, 330

Komatsu, E., \& Seljak, U. 2002, MNRAS, 336, 1256

Lagache, G., Bavouzet, N., Fernandez-Conde, N., Ponthieu, N., Rodet, T.,

Dole, H., Miville-Deschênes, M.-A., \& Puget, J.-L. 2007, ApJ, 665, L89 Laing, R. A., Riley, J. M., \& Longair, M. S. 1983, MNRAS, 204, 151
Leach, S. M., et al. 2008, A\&A, 491, 597

Lewis, A. 2005, Phys. Rev. D, 71, 083008

Lewis, A., \& Challinor, A. 2006, Phys. Rep., 429,

Lima, M., Jain, B., \& Devlin, M. 2009, ArXiv e-prints

Lin, Y.-T., et al. 2009a, in prep

Lin, Y.-T., \& Mohr, J. J. 2007, ApJS, 170, 71

Lin, Y.-T., Partridge, B., Pober, J. C., Bouchefry, K. E., Burke, S., Klein,

J. N., Coish, J. W., \& Huffenberger, K. M. 2009b, ApJ, 694, 992

Malte Schäfer, B., \& Bartelmann, M. 2007, MNRAS, 377, 253

Marsden, G., et al. 2009, ArXiv e-prints

McQuinn, M., Furlanetto, S. R., Hernquist, L., Zahn, O., \& Zaldarriaga, M. 2005, ApJ, 630, 643

Metzler, C. A. 1998, ArXiv Astrophysics e-prints

Motl, P. M., Hallman, E. J., Burns, J. O., \& Norman, M. L. 2005, ApJ, 623, L63

Nagai, D. 2006, ApJ, 650, 538

Nozawa, S., Itoh, N., \& Kohyama, Y. 1998, ApJ, 508, 17

Okamoto, T. \& Hu, W. 2003, Phys. Rev. D, 67, 083002

Ostriker, J. P., Bode, P., \& Babul, A. 2005, ApJ, 634, 964

Ostriker, J. P., \& Vishniac, E. T. 1986, ApJ, 306, L51

Pace, F., Maturi, M., Bartelmann, M., Cappelluti, N., Dolag, K., Meneghetti, M., \& Moscardini, L. 2008, A\&A, 483, 389

Patanchon, G., et al. 2009, ArXiv e-prints

Peel, M. W., Battye, R. A., \& Kay, S. T. 2009, ArXiv e-prints

Perotto, L., Bobin, J., Plaszczynski, S., Starck, J. ., \& Lavabre, A. 2009,

ArXiv e-prints

Pfrommer, C., Enßlin, T. A., Springel, V., Jubelgas, M., \& Dolag, K. 2007, MNRAS, 378, 385

Reichardt, C. L., et al. 2009, ApJ, 694, 1200

Righi, M., Hernández-Monteagudo, C., \& Sunyaev, R. A. 2008, A\&A, 478, 685

Roncarelli, M., Moscardini, L., Borgani, S., \& Dolag, K. 2007, MNRAS, 378,1259

Sadler, E. M., Ricci, R., Ekers, R. D., Sault, R. J., Jackson, C. A., \& de Zotti, G. 2008, MNRAS, 385, 1656

Santos, M. G., Cooray, A., Haiman, Z., Knox, L., \& Ma, C. 2003, ApJ, 598, 756

Schlegel, D. J., Finkbeiner, D. P., \& Davis, M. 1998, ApJ, 500, 525

Sehgal, N., Trac, H., Huffenberger, K., \& Bode, P. 2007, ApJ, 664, 149

Shaw, L. D., Holder, G. P., \& Bode, P. 2008, ApJ, 686, 206

Sheth, R. K., \& Tormen, G. 1999, MNRAS, 308, 119

Smith, K. M., et al. 2008, ArXiv e-prints

Smith, R. E., et al. 2003, MNRAS, 341, 1311

Smoot, G. F. 1998, ArXiv Astrophysics e-prints

Sokasian, A., Gawiser, E., \& Smoot, G. F. 2001, ApJ, 562, 88

Song, Y.-S., Cooray, A., Knox, L., \& Zaldarriaga, M. 2003, ApJ, 590, 664

Sun, M., Voit, G. M., Donahue, M., Jones, C., Forman, W., \& Vikhlinin, A 2009, ApJ, 693, 1142

Sunyaev, R. A., \& Zeldovich, Y. B. 1970, Comments on Astrophysics and

Space Physics, 2, 66

Sunyaev, R. A., \& Zeldovich, Y. B. 1972, Comments on Astrophysics and Space Physics, 4, 173

Toffolatti, L., Argueso Gomez, F., de Zotti, G., Mazzei, P., Franceschini, A., Danese, L., \& Burigana, C. 1998, MNRAS, 297, 117

Trac, H., Bode, P., \& Ostriker, J. P. 2009, in prep

Vallinotto, A., Das, S., Spergel, D. N., \& Viel, M. 2009, ArXiv e-prints

Verde, L., Heavens, A. F., \& Matarrese, S. 2000, MNRAS, 318, 584

Viero, M. P., et al. 2009, ArXiv e-prints

Vikhlinin, A., Kravtsov, A., Forman, W., Jones, C., Markevitch, M., Murray,

S. S., \& Van Speybroeck, L. 2006, ApJ, 640, 691

Vishniac, E. T. 1987, ApJ, 322, 597

White, M., Hernquist, L., \& Springel, V. 2002, ApJ, 579, 16

Wik, D. R., Sarazin, C. L., Ricker, P. M., \& Randall, S. W. 2008, ApJ, 680, 17

Willott, C. J., Rawlings, S., Blundell, K. M., Lacy, M., \& Eales, S. A. 2001, MNRAS, 322, 536

Wilman, R. J., et al. 2008, MNRAS, 388, 1335

Windhorst, R. A., Fomalont, E. B., Partridge, R. B., \& Lowenthal, J. D. 1993, ApJ, 405, 498

Wright, E. L., et al. 2009, ApJS, 180, 283

Yoo, J., \& Zaldarriaga, M. 2008, ArXiv e-prints, 805

Zahn, O., Zaldarriaga, M., Hernquist, L., \& McQuinn, M. 2005, ApJ, 630, 657 